\title{
Gossamer-1: Mission Concept and Technology for a Controlled Deployment of Gossamer Spacecraft
}

\author{
Patric Seefeldt ${ }^{\mathrm{a}}$, Peter Spietz ${ }^{\mathrm{a}}$, Tom Sproewitz ${ }^{\mathrm{a}}$, Jan Thimo Grundmann ${ }^{\mathrm{a}}$, Martin Hillebrandt ${ }^{\mathrm{b}}$, Catherin Hobbie ${ }^{\mathrm{a}}$, \\ Michael Ruffer ${ }^{\mathrm{c}}$, Marco Straubel ${ }^{\mathrm{b}}$, Norbert Tóth ${ }^{\mathrm{a}}$, Martin Zander ${ }^{\mathrm{b}}$ \\ ${ }^{a}$ German Aerospace Center (DLR) - Institute of Space Systems, Robert-Hooke-Str. 7, 28359 Bremen, Germany \\ ${ }^{b}$ German Aerospace Center (DLR) - Institute of Composite Structures and Adaptive Systems - Composite Design, Lilienthalplatz 7, 38108 \\ Braunschweig, Germany \\ ${ }^{c}$ Julius-Maximilians-University Wuerzburg, Computer Science 8 - Aerospace Information Technology
}

\begin{abstract}
Gossamer structures for innovative space applications, such as solar sails, require technology that allows their controlled and thereby safe deployment. Before employing such technology for a dedicated science mission, it is desirable, if not necessary, to demonstrate its reliability with a Technology Readiness Level (TRL) of six or higher.

The aim of the work presented here is to provide reliable technology that enables the controlled deployment and verification of its functionality with various laboratory tests, thereby qualifying the hardware for a first demonstration in low Earth orbit (LEO). The development was made in the Gossamer-1 project of the German Aerospace Center (DLR).

This paper provides an overview of the Gossamer- 1 mission and hardware development. The system is designed based on the requirements of a technology demonstration mission. The design rests on a crossed boom configuration with triangular sail segments. Employing engineering models, all aspects of the deployment were tested under ambient environment. Several components were also subjected to environmental qualification testing.

An innovative stowing and deployment strategy for a controlled deployment, as well as the designs of the bus system, mechanisms and electronics are described. The tests conducted provide insights into the deployment process and allow a mechanical characterization of that deployment process, in particular the measurement of the deployment forces.

Deployment on system level could be successfully demonstrated to be robust and controllable. The deployment technology is on TRL four approaching level five, with a qualification model for environmental testing currently being built.
\end{abstract}

Keywords: Gossamer-1, gossamer structures, deployable structures, solar sailing, controlled deployment

\section{Introduction}

In the last few years, the German Aerospace Center (DLR) has pursued the further development of scalable deployment technology for gossamer spacecraft structures, suitable for autonomous and controlled deployment. A summary of those developments is given in this paper. While a focus was on solar sails and thin-film photovoltaics, the aim of the development is to provide scalable technology for deployable membrane structures for various space applications. The development was made within DLR's Gossamer-1 project. The project was initiated with the goal of developing the required deployment technology and demonstrating it in low Earth orbit (LEO). It was not intended to build a full-size and functional solar sail. An artist's rendering of this demonstrator is provided in Figure 1.

The development of solar sail technology in Europe and specifically at the DLR goes back to the 1990s

\footnotetext{
${ }^{1}$ System and Mechanisms Engineer, patric.seefeldt@dlr.de
}

when the first solar sail breadboards were tested using a $20 \mathrm{~m} \times 20 \mathrm{~m}$ sail in a joint DLR, NASA/JPL and ESA project, followed by several development projects like ODISSEE (Leipold et al. (1999)) and GEOSAIL (Agnolon $(2008))$. The ground demonstration is presented by Leipold et al. (2003) and the study activities are summarized by Leipold et al. (2006).

Gossamer-1 employs the knowledge gained from these projects and reuses the previously developed carbon fiber reinforced polymer (CFRP) booms, as well as state of the art aluminum coated polyimide foil. With respect to the deployment and the evolved mechanisms, it was recognized that previous strategies had disadvantages with respect to controlling and automatizing the deployment. In addition, previous projects aimed for the realization of a complete solar sail mission with a scientific payload. This increased mission complexity and cost. Ultimately, those earlier projects were not able to realize such a mission. In consequence, a step-wise development purely focusing on the deployment technology was then pursued starting with Gossamer-1. It is a low cost technology demonstrator 


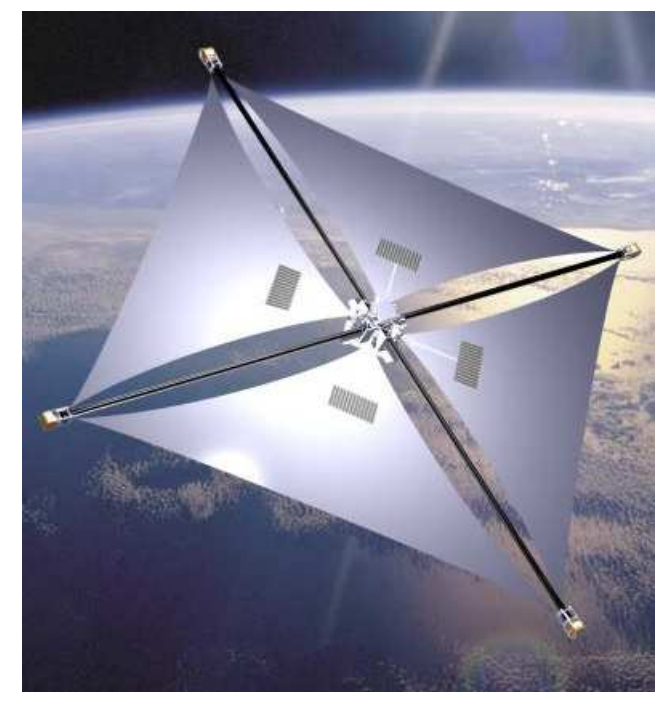

Figure 1: Gossamer-1 demonstrator, artist's rendering.

that is part of an intended three-step scalable technology development covering membranes, booms, photovoltaics and their corresponding mechanisms. Scalable means that Gossamer- 1 is a $5 \mathrm{~m} \times 5 \mathrm{~m}$ technology demonstrator using technology that is suited to build Gossamer-2 with $25 \mathrm{~m} \times 25 \mathrm{~m}$ and Gossamer-3 with $50 \mathrm{~m} \times 50 \mathrm{~m}$.

Gossamer-1 is based on a crossed boom configuration with four sail segments. At the geometric center of the spacecraft, the booms' crossing point, the central unit (Central Spacecraft Unit, CSCU) carries the satellite's main bus system, including all electronics covering command and data handling, power system, as well as ground communications system. Four Boom and Sail Deployment Units (referred to as BSDUs or deployment units) are mounted on the booms, one on each boom. In the stowed configuration, they are mechanically locked and electrically (power and data) connected to the central unit. For deployment, the deployment units are unlocked and disconnected from the central unit and move outward, thereby simultaneously deploying the booms and the sail segments. During deployment, communication with the central unit is achieved via a wireless on-board communications system, and each will have its own power system and on-board computer, as there are no wired connections foreseen in the booms. By this, a controlled and automatized deployment is realized that contrasts to the achievements of other projects like JAXA's IKAROS (see Tsuda et al. (2013)) and NASA's NanoSail-D (see Johnson et al. (2010)). The deployment process is monitored by analyzing various characteristics and can be stopped and resumed at any time, if required.

The satellite has an estimated mass of about $30 \mathrm{~kg}$ and the compact launch configuration shown in Figure 2(a) has a maximum width of approximately $790 \mathrm{~mm}$ and a height of $500 \mathrm{~mm}$. In addition, Figure A.25 provides an overview of the system components. It was planned to launch the
Gossamer-1 satellite as secondary payload in the framework of the EC FP7 Project QB50 (Thoemel (2014)). However, due to prioritization of other competing projects, it was not possible to build the complete satellite and as a consequence the launch opportunity with QB50 could not be used. What was achieved was to design a technology demonstration mission and invent a new deployment strategy that meets the above stated goals. Engineering models of all hardware were built and subjected to various tests.

In Section 2, an introduction to the intended technology demonstration mission, for which the Gossamer-1 hardware was designed, is provided. This includes some information about the programmatic environment of the project. After outlining the mission, the satellite's hardware is described in detail starting with the bus system in Section 3 . With these first sections, the mission background and the satellite bus, which hosts the deployment technology, are explained. The deployment technology is then described in Section 4. The tests conducted with the engineering models of the deployment technology are presented in Section 5. The tests show the functionality of the Gossamer-1 deployment technology, including mechanisms and electronics.

\section{Mission design}

This section provides an overview of the most important requirements and the system compliance for a deployment demonstration mission (see Subsections 2.2 to 2.6). The resulting mission phases are described in Subsection 2.7

\subsection{Programmatic aspects}

Driven by a limited budget and being a technology demonstrator, the reflective sail technology is in principle suitable for solar sailing, but it will neither have sufficient size nor an attitude control system needed for functioning as solar sail. The sail system of Gossamer-1 includes a flexible thin film photovoltaic experiment as a functional demonstrator, but this is not part of the nominal power system nor is it sized accordingly. Consequently, regarding the photovoltaics experiment, no requirements on attitude towards the sun are imposed.

As Gossamer-1 has not been among the high priority DLR projects, it had to be realized under low budget restrictions. In particular, this included a challenging low staffing plan with roughly 27 person years for the total duration of the project. For early project phases, approx. 6 person years per year on average were earmarked and available. For phases $\mathrm{C}$ and D, up to approx. 9 to 10 person years per year were temporarily foreseen. However, due to the prioritization of other competing projects, even the earmarked staffing was never realized and development had to be done with significantly less than the manpower planned. At the same time, most technical domains were staffed only part time, with engineers being also engaged with higher priority in other projects. Delays and complications in Gossamer-1 development were unavoidable. 


\subsection{Orbit}

The technology demonstration shall take place in a low Earth orbit with an initial perigee of no less than $350 \mathrm{~km}$. This is driven by increased atmospheric drag at lower altitudes and the risk of mechanical destruction of the sail and boom structure by the drag forces. The apogee shall be no more than $800 \mathrm{~km}$ to avoid increased radiation at higher altitudes. In this orbit, drag dominates the solar radiation pressure. As a deployment demonstrator, Gossamer-1 will not function as a solar sail. Consequently, it is not required to launch into altitudes where solar pressure is the dominant force. The Gossamer-1 structure, especially the sail, is suitable to operate in these altitudes.

The orbit shall have an inclination greater than $50^{\circ}$ in order to use either the ground station at Weilheim (latitude approx. $48^{\circ} \mathrm{N}$ ) or Neustrelitz (latitude approx. $53^{\circ} \mathrm{N}$ ) operated by DLR's German Space Operations Center (GSOC). An inclination greater than $75^{\circ}$ would enable more frequent ground contacts by employing high-latitude ground stations that are accessible for GSOC. That would enable a more efficient operations concept for the mission. Apart from that, the mission is designed such that it is compliant with arbitrary initial orbit orientations and drift rates in order to facilitate acquisition of a secondary payload flight opportunity.

The QB50 mission launch (see Subsection 2.3) is compliant with those orbit specifications. The technical design of the power, thermal and communication subsystems directly extends from that mission design.

The orbit shall be compliant with the space debris mitigation requirements according to ISO24113 (2011). It is ensured that Gossamer-1 will reenter by natural orbit decay within 25 years (also if the sail is not deployed). Gossamer1 is mainly a demonstrator for solar sail technology, where the ratio of total mass to sail area is the driving factor for the performance of a solar sailcraft. By this reasoning, the deployment mechanisms will be jettisoned after deployment, as they do not serve the solar sail purpose. Jettisoning those mechanisms is therefore considered a mission critical element. It is done in a sufficiently low orbit, so that the orbit lifetime of the jettisoned mechanisms is on the order of weeks.

\subsection{Launcher}

In the usual process, a satellite is designed to withstand the mechanical loads it is subjected to by the selected launch vehicle. However, for a secondary payload that has to maintain launch vehicle flexibility throughout the design process, this procedure needs to be advanced in order to enable multiple possible launch vehicles. A virtual launch vehicle was defined with a launch load envelope that results from a launch vehicle survey based on the planned launch of Gossamer-1 within the framework of the project QB50. The survey considers the launchers Cyclone-4, Dnepr, Shtil (-1), Shtil-2R, Shtil-2.1, VEGA, Falcon-9, PSLV (including PSLV-CA, PSLV-XL), Eurockot Rokot-KM, Ariane 5
ASAP (micro and mini) and Soyuz ASAP-S (micro and mini). The launch loads of the virtual launch vehicle are given in Appendix B. For Gossamer-1 the loads to be considered for qualification testing are 1.25 times the loads for acceptance testing, or the acceptance power spectral intensity $+3 \mathrm{~dB}$. The structure and mechanisms were designed according to these loads (see Subsection 3.4). Qualification testing of a deployment unit is planned (see chapter 6).

\subsection{Ground segment}

DLR's German Space Operations Center GSOC is programmatically required to be used as the ground segment. Consequently, compatibility with GSOC's procedures and technology must be ensured. For this reason compliance with the Consultative Committee for Space Data Systems (CCSDS) standards was required and implemented.

For Gossamer-1 mission objectives, S-band up- and downlink is sufficient, which is provided by GSOC ground station Weilheim.

It is intended to operate the spacecraft in normal operations with a single ground station in order to reduce cost of operations.

\subsection{Controlled deployment}

The deployment process of Gossamer-1 shall be performed in a controlled manner. This requirement refers to the fact that the whole deployment process must enable Failure Detection, Isolation and Recovery (FDIR). It requires the possibility of obtaining system status information as well as possibilities for reacting to certain system states, which in fact is the definition for controlled deployment. For this reason, the deployment strategy ensures that the system is always in a mechanically stable configuration (see Subsection 4.2 and 4.3.4) and the progress of the deployment is monitored (see Subsection 4.4).

The deployment of the Gossamer-1 demonstrator shall be performed autonomously by the spacecraft after having been initialized by a ground command. This is based on the fact that the deployment process is conducted on a time scale of some minutes while ground control is limited to available ground contacts that take place on significantly larger time intervals. Any means of near real time control are considered to be beyond the scope of the resource-limited Gossamer-1 project. The deployment logic was correspondingly implemented in the software.

\subsection{Visual documentation}

The deployment process shall be documented by means of video or image sequences and images. This enables the evaluation and documentation of the functionality of Gossamer-1 as a technology demonstrator, and to provide key data as a basis for future missions. The documentation strategy has to be optimized regarding data volume as well as accessibility of visual data for ground control in view of the S-band communications considered, possible 


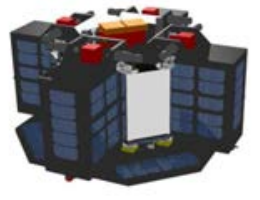

(a) Launch configuration

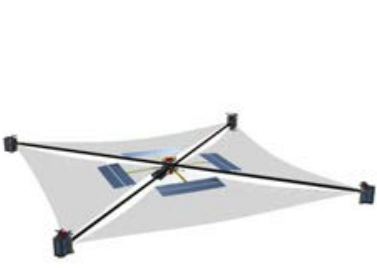

(c) Deployed

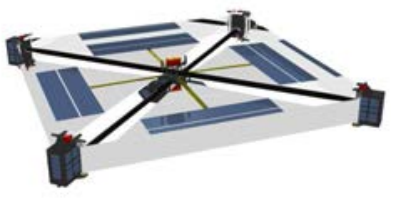

(b) During deployment

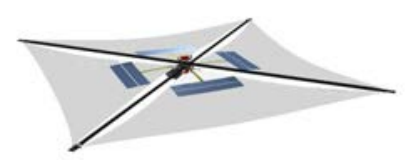

(d) Deployed and mechanisms jettisoned

Figure 2: Gossamer-1 deployment sequence.

short mission lifetime at low altitudes and FDIR strategies. Therefore, a camera system (see Subsection 4.4.2) was implemented, taking images according to predefined patterns based on the deployment process (e.g. one image every $2.5 \mathrm{~cm}$ of boom deployment distance) and not a time-based video capture with certain frames per second.

\subsection{Mission phases}

The nominal duration of the considered technology demonstration mission is on the order of 8 to 10 weeks. This duration is required in order to downlink the data generated during the deployment and to observe the system in space environment for some time. The deployment itself will only take about 10 minutes.

Figure2 shows the deployment sequence of the Gossamer1 mission. Starting with the compact launch configuration shown in Figure2(a), the deployment of the sail (Figure $2(\mathrm{~b})$ is generally finished with the configuration shown in Figure 2(c), At this point in the deployment, the sails are already separated from the sail spools and only mounted to a fixation ring that is locked on the boom. An optional jettisoning of the deployment units would be possible (see above). However, it is understood that this option is only applicable to orbits where no long-lived space debris is generated. Figure $2(\mathrm{~d})$ shows the sail craft after jettisoning.

Encompassing and incorporating this purely deploymentrelated process, the mission is subdivided into 9 phases (see Figure 3), which take mission operation considerations into account. The following paragraphs describe the phases in further detail.

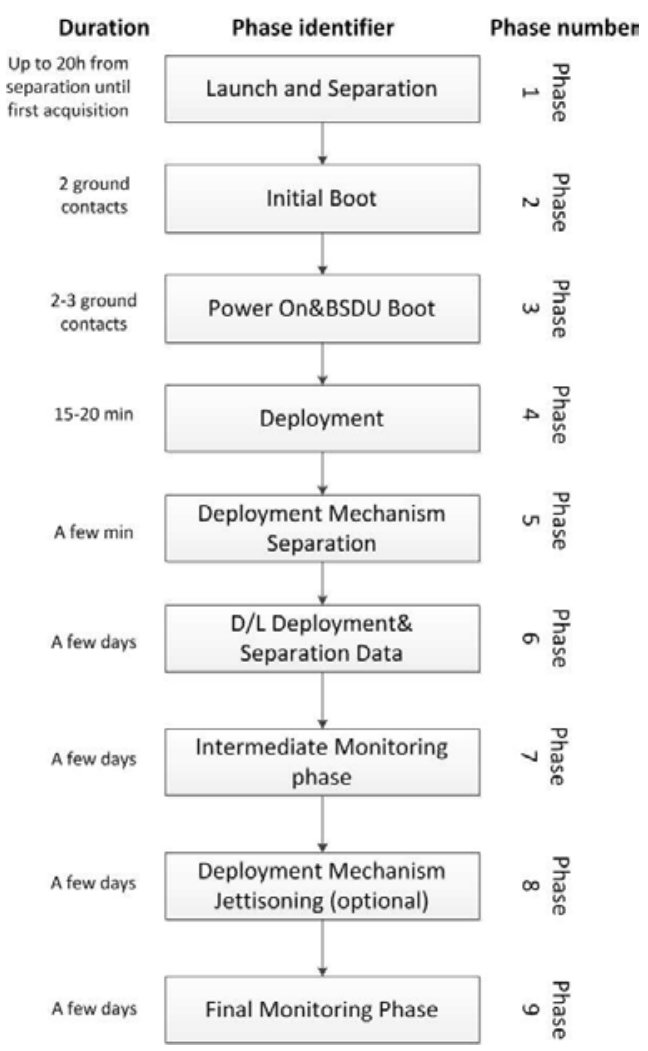

Figure 3: Gossamer-1 mission phases.

\subsubsection{Phase 1: launch and separation}

During launch and separation from the launcher, the spacecraft is in the stowed configuration as shown in Figure 2(a). At separation, the spacecraft is activated by a launcher separation switch.

\subsubsection{Phase 2: initial boot}

In the initial boot process, the spacecraft is still in the stowed configuration. The on-board computer (OBC) and the S-Band communication system are booted. This is done in a stepwise incremental procedure to allow close spacecraft control and FDIR in case of non-nominal behavior.

The first acquisition of the spacecraft by the ground segment takes place in this configuration. The spacecraft has enough autonomy and power to survive without ground contact for a sufficiently long time even if problems occur in the first acquisition. It should be noted that this is a statement about the space segment only, not about spacecraft acquisition and the decreasing accuracy of orbit propagation in case of prolonged failure in first acquisition.

The camera system on the central unit is then booted up and the first test images are taken. Status information and test images are downlinked. This phase is expected to be covered with two ground contacts. 


\subsubsection{Phase 3: BSDU power on and boot-up}

Throughout this phase, the spacecraft still remains in stowed configuration. After the central unit has been booted up successfully, the deployment units are switched on and booted one by one. The on-board wireless communications system is initialized and connections between the central unit and all deployment units will be tested one by one. After that, all five power systems (one on the central unit and one in each of the four deployment units) and their charging networks are configured to provide an optimum initial state of charge for each unit's battery.

The cameras on the deployment units are booted up, test images are acquired and sensors are read out. All of these data are subsequently downlinked to ground. Based on on-ground analysis, reconfiguration or calibration of sensors as well as camera exposure time reconfiguration can be considered.

For this power on and boot phase, roughly two to three ground contacts are expected to be necessary. The length of this phase is driven by the ground contact schedule, and not by the length of space segment activities.

\subsubsection{Phase 4: deployment}

The deployment phase is started via a time tagged command to ensure that deployment starts in sunlight and just prior to the next downlink such that nominal deployment (which consists of Phase 4 and 5, see below) will be done a few minutes before the actual ground contact. It is desirable to start the deployment in sunlight in order to have good illumination for camera monitoring of the deployment. The deployment starts just prior to the next downlink in order to ensure that in the case of nonnominal behavior, the system status is quickly known via status downlink. By that, apart from autonomous onboard FDIR, ground commanded FDIR measures can also be considered and realized.

When the deployment is started, the deployment units with the sail spool mechanisms are unlocked and the units move outward, deploying the booms and the sails at the same time, see Figure 2(b). Communication between them and the central spacecraft unit will be done via the onboard wireless communication system.

Throughout this whole phase, images are taken by all 9 cameras, 5 on the central unit and one on each deployment unit. Likewise, data about position and moving speed of deployment units, deflection of booms as well as related house keeping data is acquired for control of the deployment process. In case of non-nominal behavior, the system autonomously stops the deployment (emergency stop) and transits into a safe mode. Different cases for emergency stops are defined. In the case of such an emergency stop, all sensor data is read out and all cameras will take high resolution images. Such emergency stop data packages and corresponding historical data are subsequently downlinked with first priority at the next ground contact.

In the nominal case, the deployment units will stop at the nominal deployment's end position and one part of the deployment unit, the boom-sail interface, will lock into the boom.

\subsubsection{Phase 5: deployment units separation}

Under nominal conditions, the spacecraft continues into this phase autonomously. As the next step of deployment, the deployment units mechanically separate from the boom-sail interfaces and move a few centimeters away from the fixation point of the boom-sail interface while still remaining on the outer end of the booms. Before and after this separation, all cameras take high resolution images. After successful separation, image data, housekeeping data and science sensor data are then transferred from the deployment units via the on-board wireless communication system to the central unit, where they are stored for downlink.

This separation phase does not include a ground contact. Its duration is on the order of a few minutes.

\subsubsection{Phase 6: downlink of deployment and separation data}

The next step is to download all data safely to the ground station. Image data amounts to roughly 300 MBytes for nominal deployment without housekeeping data and coding overhead. Assuming a 1 Mbps download this would require less than 10 ground contacts. Between downlinks, the system enters a standby mode in which it autonomously performs ground contacts and science measurements (photovoltaics experiment, deflection measurements on booms) according to a predefined schedule.

The duration of this phase is driven by the required number of ground contacts for downlink. If only Weilheim or Neustrelitz and only day shifts were used, this would correspond to a few days.

\subsubsection{Phase 7: intermediate monitoring phase}

During this phase the system is mostly in standby mode. The system autonomously wakes up to take science measurements on the photovoltaics only according to a predefined schedule (e.g. transition from umbra to sunlight), as well as deflection measurements of the booms, acceleration measurements of the deployment units and magnetic field measurements for attitude determination. Sporadic recording of images is considered as an option.

The main drivers for the length of this phase are the deflection and eigenfrequency measurements under different thermal and drag conditions. These conditions change along the orbit and with the natural decay of the orbit. The duration is on the order of days.

Ground contacts are limited to what is needed to maintain the necessary orbit propagation accuracy required by the ground segment, as data volume will be low.

\subsubsection{Phase 8: BSDU jettisoning experiment}

This jettison is a central part of the solar sail use case, to shed dead mass. This is done in order to minimize the 
mass of the sailcraft and thereby maximize the characteristic acceleration, reachable with a solar sailcraft. After jettisoning all deployment units, the spacecraft is in sailcraft configuration as shown in Figure 2(d). The system reaction upon jettisoning will be observed by the cameras, strain gauges, and high sensitivity accelerometers (see Subsection 4.4).

The deployment units shall be jettisoned one by one in order to reduce the risk of collision. Furthermore, each jettison is considered a unique and individual experiment, thereby providing a chance to collect more data and also having the chance to use the remaining deployment units and their sensors for additional measurements. During the actual jettisoning, all cameras will take images in high resolution to provide information on visible boom deflections. The deployment unit being jettisoned will also take images and record data. This first stage of jettisoning is expected to last a few minutes.

When the respective deployment unit reaches a distance of a few meters, the first phase of jettisoning is ended and imaging stopped. The second phase concentrates on catching images of the spacecraft with the camera of the receding deployment units to obtain an overall view of the spacecraft. Frame rate will be adapted to increasing distance. The experiment for each individual jettisoning of a deployment unit is considered closed when it has reached a certain threshold distance or when it is out of range of wireless communication.

The length of this deployment unit drift and observation phase is expected to be on the order of hours. During the jettisoning experiment, data transfer from the jettisoned deployment units to the central unit will be prioritized. That data will be transferred to the ground in the same way as during the previous downlink phase (compare Phase 6).

The pattern described will be repeated for each deployment unit. Data volume will become smaller with each jettison as the number of monitoring cameras on the deployment units reduces one by one.

\subsubsection{Phase 9: final monitoring phase}

This phase is similar to Phase 7 , with the exception that the deployment units are no longer available. Nevertheless, boom deflections can still be measured with strain gauges mounted on the boom at the boom cross on the central unit, and external disturbing moments can still be determined via coarse position and attitude determination. The photovoltaics experiment is still fully functional and further information, e.g. about the degradation of the cells can be collected. During this phase the system is again mostly in sleep standby mode. The system autonomously wakes up to take science measurements only according to a predefined schedule. Ground contacts are limited to what is needed to maintain the necessary orbit propagation accuracy required by the ground segment, as data volume will be low.

\section{Satellite Bus and Auxiliary Systems}

The Gossamer-1 spacecraft bus system consists of a main bus system on the central spacecraft unit and four identical secondary bus systems, one on each deployment unit. The latter are fully independent and have their own On-Board Computer (OBC) and power subsystem. This is required, as the deployment concept of Gossamer-1 does not allow wired connections between the deployment units and the central spacecraft. Communication between the five units (CSCU and four BSDUs) is based on a wireless Bluetooth network where the OBC of the CSCU is the master and the BSDU OBCs (BBC) are the slaves.

Driven by programmatic limitations, Gossamer-1 had to be realized in a low cost environment. At the same time, the mission's character is that of a technology demonstrator with only very short mission duration in the order of a few weeks to maybe some months. These two factors required specific component selection criteria, specific reliability considerations and specific redundancy concepts. The low cost environment prohibited the use of high reliability components and full-blown standard space mission components. Instead, the design had to be based on affordable CubeSat electronics components and commercial off-the-shelf components (COTS). The latter were qualified in dedicated qualification tests.

The primary bus system on the central unit is based on the CLAVIS bus system developed at DLR Bremen (Spröwitz et al. (2010)). A first version of this is currently flying on DLR's AISat satellite, which was launched on June 30, 2014. Reuse of CLAVIS bus system hardware was a programmatic requirement to control costs. The Hardware of the Gossamer-1 electronics is based on the following components:

- CSCU On-board Computer (OBC) - Gomspace NanoMind $\mathrm{A} 712 \mathrm{C}$

- BSDU On-board Computer (OBC) - in-house design, STM32F40 microcontroller

- CSCU and BSDU Electric Power System (EPS) Clyde Space CS-XUEPS2-60

- CSCU Power Distribution Module (PDM) - Clyde Space CubeSat PDM

- CSCU S-Band Transceiver - IQ Wireless

- CSCU TC/TM Encoder/Decoder - AAC microtec $\mu$ RTU 312

Lower reliability on the component level of CubeSat and COTS components required specific redundancy concepts on system level. At the same time compromises regarding specific functionalities were necessary, as CubeSat and COTS components had to be used without having the possibility for adaptation and modification (due to budget limitations). The same is true for limitations dictated by the re-use requirement regarding CLAVIS electronics. 
Gossamer-1 functionalities had to be adapted to these functional limitations.

For the command and data handling of the central unit a redundancy approach was chosen based on two warm redundant $\mathrm{OBCs}$ in a worker-monitor configuration. Likewise, partial redundancy is realized by the use of two PDMs. Redundancy is only partially realized, as the number of switches per PDM is not sufficient to provide redundancy for all switched functionalities. For a subset of all functionalities, it had to be accepted that in the case of the failure of one PDM, only the prioritized functions realizable with one PDM can be maintained. All others will have to be switched off in that case.

As only limited data bus functionality is available with the NanoMind OBC as well as PDMs, the worker-monitor network had to be realized with an $\mathrm{I}^{2} \mathrm{C}$ data bus, which is known to be less reliable than others. At the same time, due to the limited number of data bus interfaces, it was necessary to also connect the PDMs to the worker-monitor network, even though failure of components (nodes) in the $\mathrm{I}^{2} \mathrm{C}$ network would cause the failure of the $\mathrm{I}^{2} \mathrm{C}$ bus as such, and thereby failure of the crucial monitor-worker network. To compensate for that, two parallel $\mathrm{I}^{2} \mathrm{C}$ networks were implemented plus the installation of $\mathrm{I}^{2} \mathrm{C}$ buffers at each node, allowing the disconnection of faulty components from the bus, thereby protecting the $\mathrm{I}^{2} \mathrm{C}$ bus from corruption by faulty components. In addition, suitable hierarchies of watch dog and timeout cascades were implemented.

For the Bluetooth system, two parallel central node networks are installed, each of which is connected to one OBC, each with its own internal redundancy. This was also driven by the limited availability of interfaces, but fortunately relaxed by low cost, low mass and low power consumption of Bluetooth COTS components. To increase reliability, Bluetooth modules are cross-switched by the two PDMs, i.e. of each pair of Bluetooth modules per OBC one is switched by one PDM, and the other by the other PDM. On the BSDUs, space and power are severely limited. Therefore no OBC redundancy is implemented. For Bluetooth, two cold redundant modules are installed.

For the whole Gossamer-1 bus system, see Figure C.30

\subsection{Power subsystem}

Gossamer-1 has power systems on the central unit and one on each deployment unit. All five power systems are based on the same electrical structure. As Figure 4 shows, this includes individual solar cells, batteries, an electronic power system and a power distribution module for each. In stowed launch configuration, they are not connected to each other at first. After the successful boot-up of all five power systems, when the nominal state is verified for each of them, they will be interconnected to form a charging network to provide an optimum initial state charge for each unit. In a non-nominal case, if considered necessary, a charging phase can be introduced in which wellilluminated units autonomously recharge shadowed units before normal operation is resumed.
The dimensioning of power systems assumes a worst case eclipse duration of 37 minutes applicable for all low Earth orbits in the considered altitude range and a ratio of $41 \%$ eclipse, including penumbra, and $59 \%$ fully sunlit, applicable for very low-altitude orbits.

All five power systems rely on standard photovoltaic cells used for CubeSats, either Azur 3C30 or Spectrolab, as operational photovoltaics. To enable high area efficiency, a mix of standard- and small-size cell equipped strings is used; e.g. $(2 \mathrm{~cm})^{2}$ cells are used on CubeSat small panels and on the asteroid lander MASCOT which was integrated at DLR Bremen (see Ho et al. (2016)).

The central unit will hold eight groups and each deployment unit six groups of strings of 6 to 8 triple-junction cells each. Under full illumination, one string of standardsize triple-junction cells operates at $7.15 \mathrm{~W}$ rated power at $+80^{\circ} \mathrm{C}$. In addition, Gossamer- 1 demonstrates the use of thin film photovoltaics in space by realizing a Thin Film Photovoltaics Experiment located on the inner part of the four sail segments (see blue rectangles in Figure 21). The experiment is patched into the power system, but the operational photovoltaics are designed such that energy requirements are met with the standard photovoltaics alone. The photovoltaics experiment is considered as an experimental add-on and consequently not required for any other system operations.

ABSL was chosen as a battery supplier. The ABSL $18650 \mathrm{HCMs}$ are self-balancing cells with two independent safety devices, a Positive Thermal Coefficient (PTC) reversible device for overcurrent and temperature and a Circuit Interrupt Device (CID, irreversible) for overpressure. The use of stacks with two cells connected in a series and those connected six times in parallel (2S6P) are foreseen. One stack will be employed for each deployable unit and four of these stacks are employed for the central spacecraft unit. It is possible to upgrade to the higher capacity $18650 \mathrm{NL}$ cell, either to reduce overall spacecraft mass or increase battery margin.

The Electrical Power System (EPS) is a Clydespace CSXUEPS2-60. It provides six step-down Maximum Power Point Tracking Battery Charge Regulators (MPPT-BCR) for $12 \mathrm{~W}$ input power at $10 \mathrm{~V}$ to $25 \mathrm{~V}$ with $0.75 \mathrm{~A}$. Each regulator can connect to two photovoltaic strings. An ideal diode toward the battery prohibits reverse discharge through the photovoltaic cells and MPPT-BCRs. The power output section provides either $3.3 \mathrm{~V}$ at $4 \mathrm{~A}, 5.0 \mathrm{~V}$ at $4 \mathrm{~A}, 12 \mathrm{~V}$ at $1 \mathrm{~A}$ or $6.2 \mathrm{~V}$ to $8.2 \mathrm{~V}$ (unregulated).

The Power Distribution Module (PDM) is a Gomspace CN_SWT - $0035-$ CS. It provides 24 individually protected power switches:

- seven 3.3V, rated $0.5 \mathrm{~A}(3 \mathrm{x}), 1 \mathrm{~A}(3 \mathrm{x}), 4 \mathrm{~A}(1 \mathrm{x})$

- seven 5.0V, rated $0.5 \mathrm{~A}(3 \mathrm{x}), 1 \mathrm{~A}(3 \mathrm{x}), 4 \mathrm{~A}(1 \mathrm{x})$

- five VBat, rated $0.5 \mathrm{~A}(2 \mathrm{x}), 1 \mathrm{~A}(2 \mathrm{x}), 4 \mathrm{~A}(1 \mathrm{x})$

- five $12 \mathrm{~V}$,rated $0.25 \mathrm{~A}(2 \mathrm{x}), 0.5 \mathrm{~A}(2 \mathrm{x}), 1 \mathrm{~A}(1 \mathrm{x})$ 


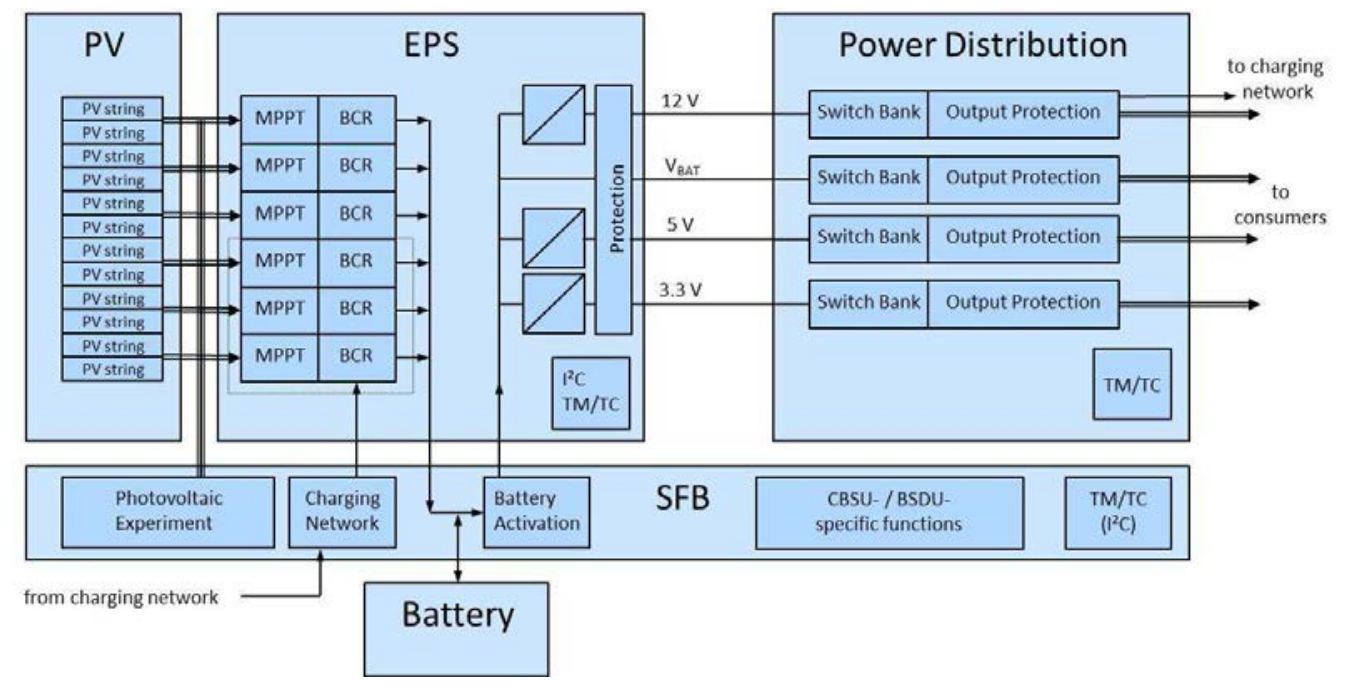

Figure 4: Power subsystem schematic (implemented five times in the Gossamer-1 system).

The PDM provides an $\mathrm{I}^{2} \mathrm{C}$ TM/TC node interface with

- 24 internal analogue telemetry channels with 10 bit $\mathrm{A} / \mathrm{D}$ conversion

- 40 external analogue telemetry channel inputs with 10 bit $\mathrm{A} / \mathrm{D}$ conversion

- 8 channels for configurable input voltages

- 32 channels for 0 to $3.00 \mathrm{~V}$ input range

- 5 serial $\mathrm{Rx} / \mathrm{Tx}$ ports for standard data rates of 300 to 115200 baud, 8 bit data format, selectable parity, and data buffers; hardware-configurable for LVTTL, TTL, RS232 or RS422

\subsection{Attitude control}

Gossamer-1 does not require an active attitude control system. The preliminary analysis showed that tumbling rates after separation and with deployed sail will fall within acceptable ranges, i.e. non-destructive regarding the deployment and the deployed configuration. In addition, the deployment itself will stabilize the satellite due to the increase of the moments of inertia by the deployment units that move away from the central unit. With ongoing mission time and increasing drag, the attitude of the deployed sail might get more unstable, but controlling the deployed configuration would require a special attitude control system that is at least beyond the mass, power and funding limitations of Gossamer-1. This would be part of the further development of the system within a Gossamer-2 project according to the stepwise development outlined in Section 1, within Gossamer-1 such development is neither planned nor funded.

\subsection{Thermal}

A preliminary analysis was conducted that considered a variety of different possible orbits. The simulations investigated the temperature change of the satellite along the orbits. The thermal control system of Gossamer-1 has to cope with highly varying conditions. Firstly, it has to guarantee the required temperature ranges under nonpredictable arbitrary orientation because Gossamer-1 will not have an attitude control system. It is only possible to estimate the spin rates. Secondly, due to the mission as such, the thermal control system has to cope with three significantly different configurations, which are:

- stowed configuration,

- deployment phase and

- deployed configuration.

These three phases will differ greatly in geometric (and thermally relevant) configuration, as well as in overall power consumption and in distribution of electrical and thermal load as a function of time due to strongly varying activity patterns. Thermal control will rely on passive elements like Multi-layer and Single-layer insulation (MLI and SLI), thermal coatings, heat conduction elements and radiators. The only active elements will be heaters for control of the cold case.

Within the central unit, the electronics compartment (CLAVIS box, see Figure A.25) contains the largest heat source. The electronics compartment is made from aluminum and heat will be transferred via the central aluminum structure to the launch adapter cone, which also functions as radiator. This provides a rotationally symmetric field of view for radiation with respect to rotation around the sail normal axis. In the deployed configuration, this will be the spacecraft's most likely spin axis. Therefore 
such a rotationally symmetrical radiator arrangement provides the largest possible independence from unpredictable attitude and spin rates.

In contrast to the central unit, the deployment units have to be considered as honeycomb Carbon Fiber Reinforced Plastic (CFRP) boxes with localized heat sources within, i.e. some electronic boxes, the motor as well as the battery stack. Heat distribution inside the deployment units is achieved by a suitable combination of SLI and thermal coatings, thereby enabling homogeneous radiative distribution of heat. Conductive paths through the honeycomb CFRP walls will propagate heat to the outside, where again a w.r.t. the sail normal rotationally symmetric radiator arrangement (circumferential metal foil radiator) will provide a large degree of independence from attitude. Also, with a relatively large fraction of their surface and most sides covered by photovoltaic cells, the BSDUs resemble the generally benign radiation balance of CubeSats more than the CSCU.

\subsection{Structure}

An overview of the overall Gossamer-1 assembly is provided in Figure A.25. The structural parts of the CSCU are the CSCU Camera Mast, the CSCU Boom and Sail Mast, the CSCU Structure, the CSCU Adapter Plate and the CSCU Launch Adapter Cone. The CSCU structural elements are mainly CFRP parts utilizing additional standard elements such as sandwich plates with aluminum honeycomb core and various types of rods. The electronic CLAVIS box and the adapter cone are made of aluminum.

The BSDU structure is constructed out of several composite sandwich plates. Those plates form a rectangular closed box. The plates are mounted to each other using screws and inserts at overlapping edges. The sandwich is composed of plates $10 \mathrm{~mm}$ thick with an aluminum honeycomb cores and quasi-isotropic CFRP face sheets. Optional uni-directional CFRP layers for local reinforcement of the face sheets are employed.

A structural Finite Element Method (FEM) analysis was implemented. The model of the CSCU Structure, the CSCU Launch Adapter Cone, the CSCU Adapter Plate and the CSCU Boom and Sail Mast (see Figure A.25 consist of shell elements (QUAD4, few TRIA3), except for the struts that attach the outer BSDU interfaces, which are bar elements (BAR2). Interfaces and screw connections are modeled using multi-point constraints (MPC, type RBE2) which connect certain degrees of freedom (DOF) of one or more dependent nodes to a single independent node. They have been used to model screw connections between the flange of the CSCU Launch Adapter Cone and the flange of the CSCU Structure, as well as to connect the CLAVIS box with the CSCU Adapter Plate. Within the FEM, several components, such as the Battery Boxes, are modeled with point mass elements. These elements are connected to their interface points using MPCs. The results with respect to the first three eigenfrequencies and eigenmodes are shown in Appendix D.
The lowest eigenfrequency of the structure is $107 \mathrm{~Hz}$. Besides eigenfrequencies, the structural analysis revealed amplification factors at the interfaces between overall structure, sub-components and mechanisms. These amplification factors were used for the dimensioning of the mechanisms. Instead of taking vibration loads for the mechanism design into account, which would result in FEM models for each mechanism, the decision was made to only consider a representative local static load. The maximum loads are driven by the random vibrations. The representative local static load is calculated by considering a three sigma environment of the vibration loads multiplied with the amplification factor $A_{F}$. The $G_{r m s}$ value, the one sigma value, is chosen according to the launch load envelope, and its value is $12.4 \mathrm{~g}$. The acceleration to be considered in the mechanism design is calculated according to equation [1.

$$
a_{m}=3 \cdot G_{r m s} \cdot A_{F}
$$

Table 1 provides the amplification factors for a selected set of mechanisms. Because the exact mounting position and orientation of Gossamer-1 on the launcher is also uncertain, the maximum amplification factor across all three axes was considered in the mechanism design process.

Table 1: Amplification factors for a selected set of mechanisms.

\begin{tabular}{ccccc}
\hline Mechanism & $A_{F x}$ & $A_{F y}$ & $A_{F z}$ & $A_{F \max }$ \\
\hline \hline Sail Spool & 2.56 & 3.03 & 3.36 & 3.36 \\
\hline BSDU Gear-Motor & 3.60 & 3.12 & 3.12 & 3.60 \\
\hline BSDU Electronics & 1.71 & 2.11 & 3.36 & 3.36 \\
\hline BSDU Battery Box & 1.28 & 2.39 & 2.93 & 2.93 \\
\hline CLAVIS Box & 1.22 & 1.70 & 2.47 & 2.47 \\
\hline
\end{tabular}

\section{Boom and sail deployment technology}

In the following paragraphs, the subcomponents and mechanisms enabling the deployment will be described in further detail.

\subsection{Booms}

Gossamer-1 makes use of light-weight coilable CFRP booms, also referred to as collapsible tube masts (CTM), as shown in Figure 5(a). Two booms are configured in a cross-like arrangement with a vertical displacement between them. The booms are mounted to the CSCU via a Boom-Spacecraft Interface (BSI). This is shown in Figure $5(\mathrm{~b})$.

The DLR previously developed and investigated the technology in precursor projects, e.g. ODISSEE (Leipold et al. (1999)) and Geosail (Agnolon (2008)). The cross section of the boom was chosen according to the smallest possible dimensions that allow coiling of the boom without reaching critical stress levels in the material, which would result in the delamination of the boom flanges and 
material fracture. The length of one full-diagonal boom is $8.6 \mathrm{~m}$. It is determined by the chosen sail size of $5 \mathrm{~m} \times 5 \mathrm{~m}$ and the necessary remaining length for the jettisoning of the BSDUs. The cross section of the booms is given in Figure 5(c). It shows the dimensions of the deployed and stowed configuration. The length is given in Figure 5(d)

During the deployment, the boom cross section is not constant along the boom. The diameter increases in $\mathrm{y}$ direction along the deployed length, starting with the flat configuration at the point where the boom is coiled inside the BSDU (see Figure 5(c). It takes more than one meter until the boom's cross section in y-direction is comparable to the deployed configuration and a small distortion of the cross section can be observed along the whole boom length. Due to the reduced geometrical moments of inertia, respectively bending stiffness, the boom section closest to the boom hub at which the boom is uncoiled is most sensitive to mechanical loads and therefore requires a linear guiding.

The booms are made of two CFRP half shells with an omega cross section, fabricated from a $0^{\circ} / 90^{\circ}$ one layer prepreg (LTM ${ }^{\circledR}$ 123CF140, CYTEC Industries Inc., USA), a plain weave fabric of $0.14 \mathrm{~mm}$ in thickness $\left(0^{\circ}\right.$ is defined by the longitudinal axis of the booms). These half shells are bonded together along the flanges (using Hysol ${ }^{\circledR}$ EA $\left.9359.3^{\mathrm{TM}}\right)$. Due to the limited size of the mold tool used for manufacturing, two boom segments of $4.3 \mathrm{~m}$ are bonded together in order to reach the required $8.6 \mathrm{~m}$ length of a full-diagonal boom. For the bonding additional CFRP patches are employed that are overlapping the joint. This bonding is located exactly at halfway of the full-diagonal at the BSI. Each BSI employs elastic glass fiber fleece hinges for a flexible mounting of the booms.

\subsection{Sail}

A preliminary material selection was presented by Seefeldt et al. (2014a). For the sail membranes, the $7.5 \mu \mathrm{m}$ thick polyimide foil Upilex-S ${ }^{\circledR}$ covered on both sides with $100 \mathrm{~nm}$ vacuum deposited aluminum was chosen. It is delivered on a roll with a width of $1.016 \mathrm{~mm}$. Additionally, samples were coated with silicon oxide on top of the aluminum to increase the infrared emittance. The effect of this additional coating is still under investigation. The sails are manufactured by using $3 \mathrm{M}^{\mathrm{TM}}$ transfer adhesive tape 966 . In a first step, five sheets are prepared, one with the photovoltaics. By bonding the edges with the transfer adhesive after folding them over, a reinforcement against cracks is achieved. The adhesive tape is also used to mount interfaces and attach the photovoltaics with its harness. In a final step all segments are bonded together in order to achieve the required sail size.

Within the Geosail precursor project (Agnolon (2008)), the transfer adhesive was tested for low temperatures down to $-142^{\circ} \mathrm{C}$. In addition to that work, a short term duration test in a furnace was conducted, reaching temperatures up to $230^{\circ} \mathrm{C}$. In these tests, the adhesive bonding was loaded

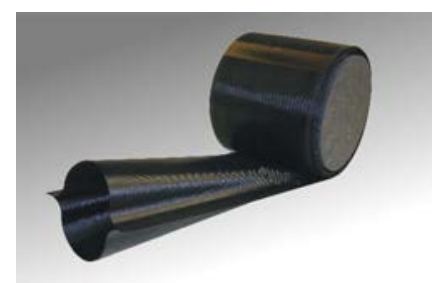

(a) Photograph of a partially stowed boom with larger cross section compared to Gossamer1.

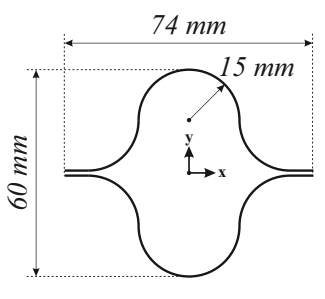

deployed

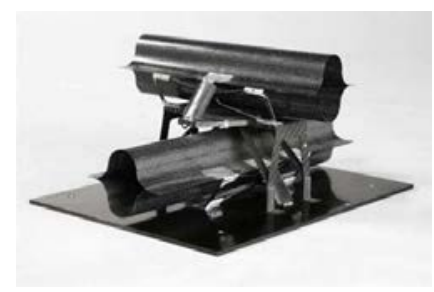

(b) Boom Spacecraft Interface for two crossed booms with vertical displacement. (c) Cross section and coordinate system definition of the Gossamer-1 boom

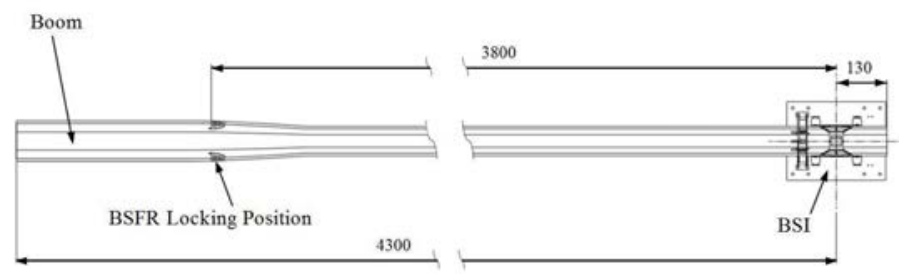

(d) Length of the boom. Note that the boom is slightly compressed at the locking position, and as consequence, it is slightly wider in the vicinity of the locking position.

Figure 5: Coilable thin shell CFRP booms and interfaces.

with $0.9 \mathrm{~N} / \mathrm{cm}^{2}$ (shear) and withstood the high temperature. The loading was roughly twice as high as the limit specified in the data sheet. In contrast to the design presented by Seefeldt et al. (2014a), a rigging is no longer used in the present design. Instead, the interface points are reinforced with a thicker copper coated foil, similar to what is used for flexible PCBs. The connection between those reinforced sail interfaces and the interfaces to the boom as well as the CSCU are made of a $0.45 \mathrm{~mm}$ stainless steel ropes.

The stowing strategy is shown in Figure 6. The triangular segments are folded in a zig-zag pattern and coiled onto two spools. The spools are mounted on two neighboring BSDUs (see Figure 2(a)). Folding and coiling the sail this way is key for controlled deployment. When the sail is deployed, only the minimal required amount of sail is uncoiled from the sail spools, such that the deployed sail is always under tension. The stowing strategy was subject to intensive testing, presented by Seefeldt et al. (2014b). The tests are summarized in Subsectior 5.2.

The photovoltaic part of the prototype sail is shown in Figure 7. The thin-film photovoltaics are located at the 


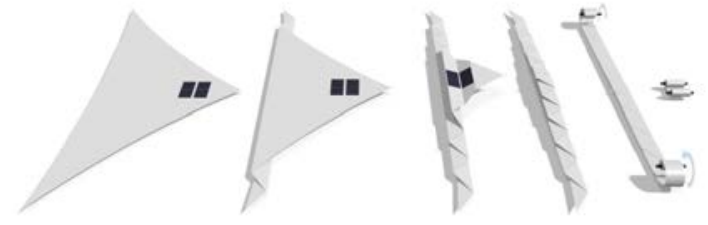

Figure 6: Gossamer-1 sail stowing strategy for one of the four segments.

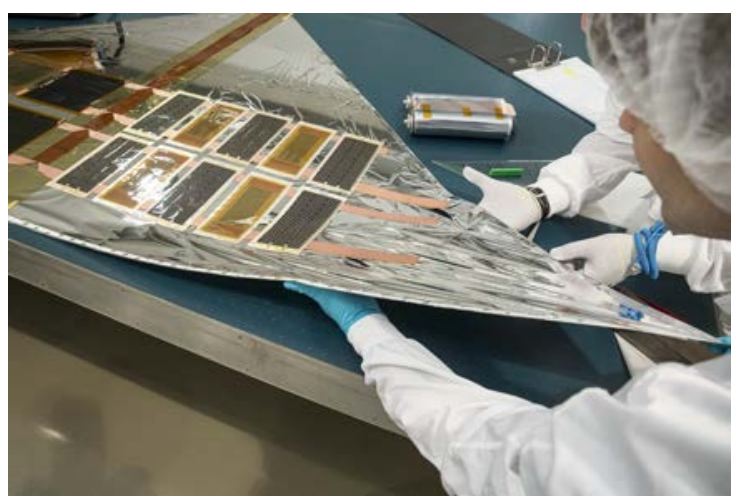

Figure 7: Thin-film photovoltaic integrated on the sail foil.

inner corner, close to the CSCU. These consist of small experimental modules that are bonded to the sails employing the abovementioned transfer adhesive. The modules are electrically contacted to a flexible PCB harness in the middle of the triangle. Note that the modules are currently experimental prototypes that are the subject of ongoing studies.

\subsection{Boom and sail deployment mechanisms}

As explained in the previous sections, the deployment is driven by BSDUs that are moving away from the CSCU. Booms and sail segments are thereby deployed at the same time. Figure 8 shows one BSDU (without the sails) during the deployment process, including the jettisoning of the BSDU. For further clarification, details of the mechanisms are shown in Figure 9. The engineering model of the boom deployment mechanism was also presented by Straubel et al. (2015).

In the stowed configuration, shown in Figure 8(a), launch locks secure the BSDU onto the CSCU (see Subsection 4.3.5). Prior to the deployment, those locks are released. The deployment is driven by an additional belt that is coiled on the boom hub together with the boom. At the very end of the boom, a small piece of Velcro connects the belt to the boom. The boom and consequently the sails are deployed by pulling-off the belt from the boom hub, thereby uncoiling the boom as well. The deploying boom pushes the BSDU away from the CBSU, which in turn uncoils the sail segments from the sail spools. During deployment (Figure 8(b)), the load is initially transferred through friction between belt and boom, and with progressing deployment more and more through the Velcro. The transferred shear load is well supported by the Velcro and the compression loads between the coiled layers also prevent premature separation of both Velcro parts by pressing both components together.

The uncoiling of the belt and thereby the deployment is driven by a belt winding mechanism (BWM) using an electric motor. To prevent uncontrolled deployment of the booms and sails, e.g. by stored elastic energy, the boom hub and the sail spools need to have a brake mechanism, described in the following dedicated paragraphs.

To achieve the jettisoning function of the BSDU, the Boom Sail Fixation Ring (BSFR) provides the interface between the outer sail corners and the boom. During sail deployment, the BSFR is attached to the BSDU and the boom runs through the ring-like shape of the BSFR. Once the sail is fully deployed (Figure 8(c) , the BSFR is locked to the boom and mechanically separated from the BSDU (Figure $8(\mathrm{~d})$. At this point the BWM drive unit can be engaged again to further deploy the remaining coiled boom, either for just a short distance (Figure $8(\mathrm{~d})$, , or afterward to move the BSDU off of the boom completely for jettisoning (Figure 8(e) and 8(f)p.

As the sail's outer corners are not attached to the BSDU but to the BSFR, the BSDU no longer has to carry sail loads. At the final stage of the deployment, the tip of the boom is deployed by transferring the deployment load purely through the Velcro. This Velcro loading applies until Figure 8(e). At that point, the Velcro passes the pulley and the Velcro loading transforms from shear loading into peel loading (compare Figure 9(a), which separates both parts without decelerating the BSDU much. The BSDU and boom are separated completely, and the BSDU maintains the previously gained kinetic energy and floats away as indicated by Figure 8(f).

\subsubsection{Belt winding mechanism - BSDU drive}

As explained above, the BWM pulls off a $0.03 \mathrm{~mm}$ thick stainless steel belt that is coiled up on the Boom Hub together with the boom. The BWM itself consists of an electrical motor, a spool with a diameter of $40 \mathrm{~mm}$ on which the belt is coiled on, and a freewheel. Figure 9(b) shows the BWM in detail. The motor, shown in light gray, is mounted on the left and with a bracket in the middle. The winding spool is located on the right, and it is directly mounted on the output shaft of the gearbox employing a feather key. The freewheel is made of a gear with pitched teeth that is part of the winding spool and an engaging copper beryllium spring plate (blue part). The belt, which is highlighted in green in Figure 9(a) is guided by a diverting pulley to the BWM spool.

The motor for the winding mechanism has to overcome torques induced by the boom hub, the boom guidance and the sail spool. Besides the required actuation torque, a further critical point is that motors used under vacuum conditions easily overheat. As a consequence, the resulting 


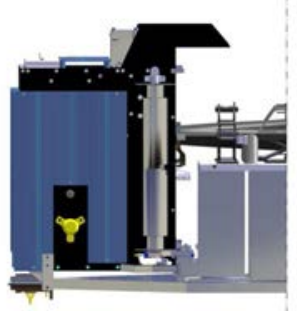

(a) Launch configuration.

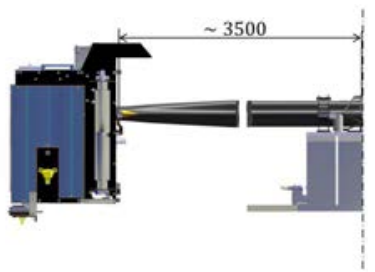

(c) The sails are fully deployed The BSFR is locked behind the yellow highlighted tape spring on the boom.

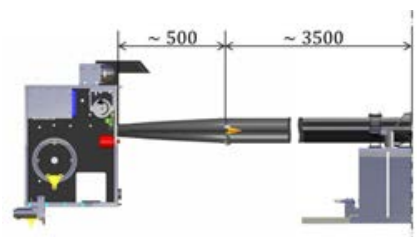

(e) The belt, connected by Velcro, is separating from the boom.

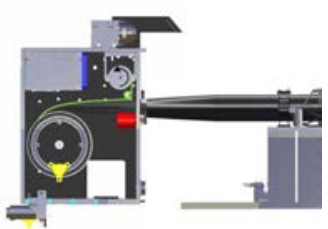

(b) BSDU is released and deployment started. The belt, highlighted in green, is coiled on BWM spool.

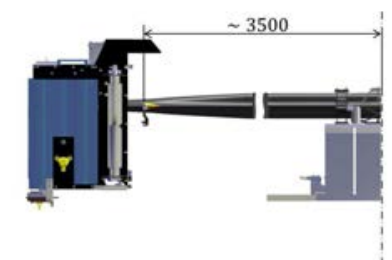

(d) The BSFR is separated and the remaining boom is deployed.

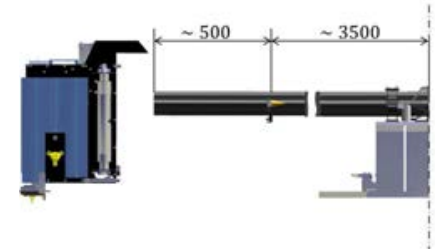

(f) The BSDU is jettisoned and drifts away.
Figure 8: BSDU deployment sequence. In order to maintain a clear representation, only one BSDU and no sail segments are shown.

possible life cycle needs to be critically evaluated. For the deployment (Phase 4) the motor has to run at a low speed but for a relatively long period of time. After a longer break for cooling down the motors during phases 5 to 7 , jettisoning will take place with high motor speed for a short period.

The Phytron VSS32 motor with a GPL32 gear box was chosen for the engineering model. For the qualification model, the company produced a customized Version of the VSS32 motor with a GPL22 gear box. In order to withstand the high mechanical launch loads, the design of motor and gearbox was made more robust. That led to a slight increase in size, which was compensated by the smaller GPL22 so that it still fits into the dedicated space inside the BSDU. The change in the gear box led to a change in the transmission ratio from 50:1 to 49:1. Under vacuum, the motor can run $6 \mathrm{rpm}$ up to $7.5 \mathrm{~min}$ for deployment, and $30 \mathrm{rpm}$ up to $1 \mathrm{~min}$ for jettison until it overheads. Considering the spool diameter of $40 \mathrm{~mm}$, the corresponding deployment speeds are $1.3 \cdot 10^{-2} \mathrm{~m} / \mathrm{s}$ for the slower mode and $6.3 \cdot 10^{-2} \mathrm{~m} / \mathrm{s}$ for the faster. It is desirable to use the slowest deployment speed possible in order to reduce inertial loads during deployment. In order to deploy the boom to $3.8 \mathrm{~m}$, the locking point of the BSFR, in the maximum motor operating time for slower speeds of $7.5 \mathrm{~min}$, the deployment speed is determined to be $0.84 \cdot 10^{-2} \mathrm{~m} / \mathrm{s}$. A torque budget, derived from force measurements with the engineering models, is given in Subsection 5.3 (Table 2).

To ensure that the belt is always under tension so that it does not slip off of the winding spool, the design includes a freewheel. This is especially required during launch, as the vibration loads might lead to a rotation of the winding spool even if the motor is turned off.

\subsubsection{Boom hub}

The boom hub consists of a rotatable mounted spool on which boom and belt are coiled. The spool is mounted with a plain bearing. The design is presented in Figure $9(\mathrm{c})$. On one side of the boom hub there is a gear with additional conical holes.

For launch, the rotation of the spool is locked by a conical pin that locks into the holes on the side of the gear. A pin puller (highlighted in yellow) releases the gear rotation in orbit. The BSDU launch lock is further described in Subsection 4.3.5.

In order to have a controlled deployment that can be stopped and resumed at any time, and to counteract the self-deployment of the boom due to stored elastic energy, the boom hub includes the aforementioned brake mechanism. It employs copper-beryllium plates (highlighted blue) that engage into the gear. Through the deformation of those leaf springs when rotating the hub, an oscillating torque with a maximum of about $0.25 \mathrm{Nm}$ is generated.

\subsubsection{Boom sail fixation ring}

The Boom Sail Fixation Ring (BSFR), shown in Figure $9(\mathrm{f})$, is the boom-sail interface.

During deployment (Phase 4), the boom slides through the BSFR. At the point where the sails are fully deployed, the sails must be mounted to the booms. This is achieved by attaching the BSFR at this position to the boom by employing tape springs that are glued onto the boom as shown in Figure 9(e), During deployment, the BSDU with the BSFR moves across the tape spring until the BSFR locks in right behind the spring.

After the ring is locked into its final position, the separation (Phase 5) between BSFR and BSDU takes place. This is achieved by employing the Ejection and Release Mechanism (ERM) E250 STD from TiNi Aerospace Inc. The mechanism is highlighted in red in Figure 9(f) It opens the mounting between BSFR and the BSDU, so that the BSDU can move further, thereby deploying the last centimeters of the booms while leaving the BSFR with the mounted sail at the fixation point. 


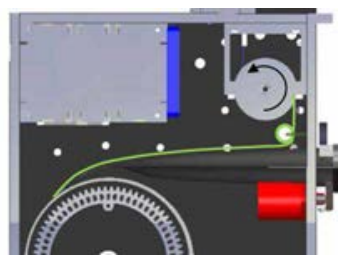

(a) View from the side. Belt (green) coiled on BWM spool (see Subsection 4.3.1.

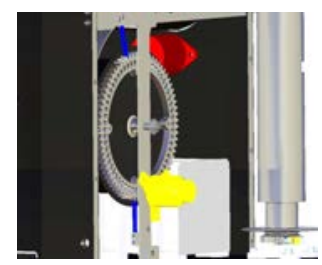

(c) Boom hub (see Subsection 4.3.2) with gear and engaging spring plates (blue). The pinpuller (yellow) can lock into holes on the side of the gear (see Subsection sec:launchlock).
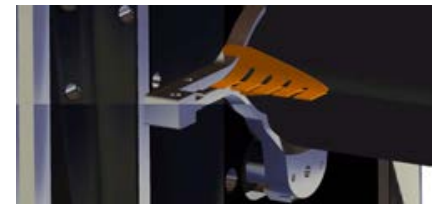

(e) Tape springs that lock the BSFR after the sail deployment is completed (see Subsection 4.3.3.

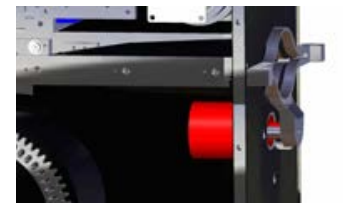

(f) BSFR and its separation mechanism (red) (see Subsection 4.3.3. (b) View from CSCU to BSDU. BWM BSDU drive and BSFR (see Subsection 4.3.3).

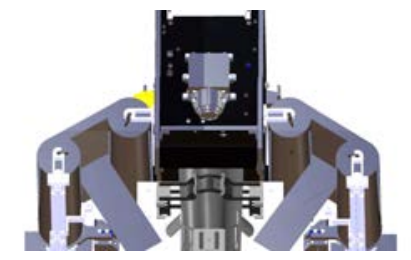

(d) View from top. Camera (see Subsection 4.4.2) mounted on the top plate and stowed sail segments on each side (see Subsection 4.3.4.

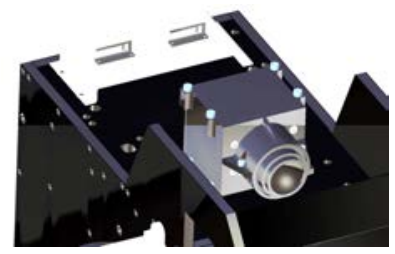

(g) BSDU top with camera (see Subsection 4.4.2) and Bluetooth antennas for the wireless communication with the main bus on the CSCU.

Figure 9: BSDU details.

\subsubsection{Sail spools}

As explained in Subsection 4.2, the sail is divided into four segments as shown in Figure 6. Each of the quadrants is folded and coiled on two sail spools, which are mounted on two adjacent BSDUs (see Figure $9(\mathrm{~d})$. An overview of the sail spool design is given in Figure 10(a). When uncoiling the sail segment, the spool with the sail interface rotates around the truss-like structure connected to the BSFR (Figure 10(b)]. After the sail segment is uncoiled, the truss-like structure directly mounts the sail through the sail interface to the BSFR (Figure 10(c)). After the separation from the BSFR (Phase 5, see Subsection 2.7), the BSDU with the sail spools drives further along the booms, thereby separating the sail spool from the truss like structure that is mounting the sail to the BSFR (Figure 10(d). The patent of Seefeldt et al. (2016) can be reviewed for details of the mechanism function.

During launch, a gear wheel on the sail spool's underside is used to lock the spool with a corresponding counterpart mounted on the CSCU. A leaf spring engaging the gear adds an oscillating break torque of approximately $0.035 \mathrm{Nm}$ at maximum. This is required during deployment to ensure that the sail does not slip off of the spool by itself. The mechanism allows a complete stop within the deployment process and still has the partly-deployed sail safely mounted.

\subsubsection{Launch locks}

During launch, all mechanisms are locked in order to provide a mechanically stable configuration of all parts. An overview of the locking mechanisms employed is provided in Figure 11.

The Boom Hub is locked using a P5 pin puller manufactured by TiNi Aerospace, Inc. Figure 9(c) shows the boom hub with the pin puller highlighted in yellow. It is mounted on the outer wall, and the pin is locked into one of the conical holes located around the boom hub gearwheel. In order to simplify the integration, 60 holes allow the hub to be locked, so that it is not necessary to reach a designated end position. Instead, the hub can be locked every $6^{\circ}$ of rotation so that after coiling the boom, the nearest locking position can be used. A cone shape for the pin and the holes was chosen in order to avoid clamping of the locked pin (e.g. due to thermal expansion).

Additionally, a form-fitting locking element ensures the locking of each sail spool by engaging its gear and a circumferential groove in the sail spool's lower end (see Figure 11 on the right and Section 4.3.4).

\subsection{Deployment Monitoring}

A first in-orbit demonstration of the deployment technology requires different sensors to gather data for the validation of the system. The system implemented a measurement of the deployed boom length presented in Section 4.4.1, a camera system shown in Section 4.4.2 and an evaluation of the boom loading with strain gauges described in Section 4.4 .3 .

\subsubsection{BSDU position determination}

The position of the BSDUs needs to be determined during deployment. Two strips with reflective markers are laminated onto the boom flanges (see Figure 12). The strips are made of a highly reflective material with transparent sections. When laminated onto the boom, the transparent sections are black due to the carbon fiber material on the backside. The material used for these strips is the 


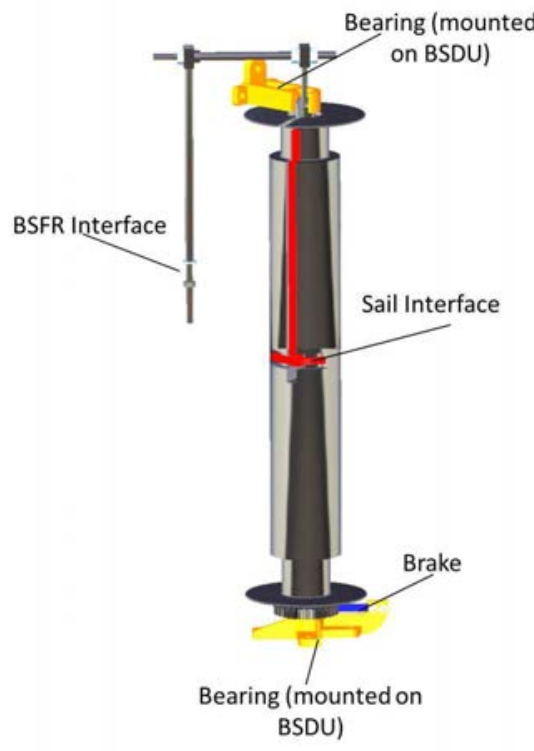

(a) Overview of the SSM components.

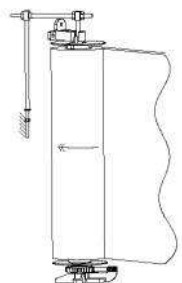

(b) Uncoiling sail segment from spool.

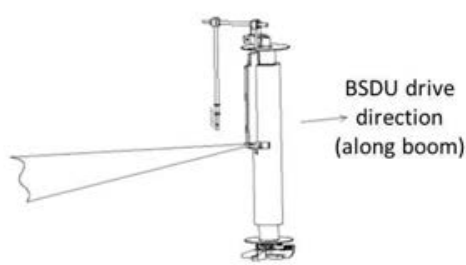

(c) Segment is uncoiled and mounted through the interface to the BSFR (which is then separated from the BSDU).

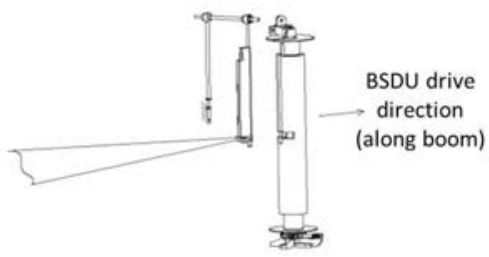

(d) The sail spool (connected to the BSDU) moves further, thereby completely separating from the interface that mounts the sail to the BSFR.

Figure 10: Sail spool and separation sequence.

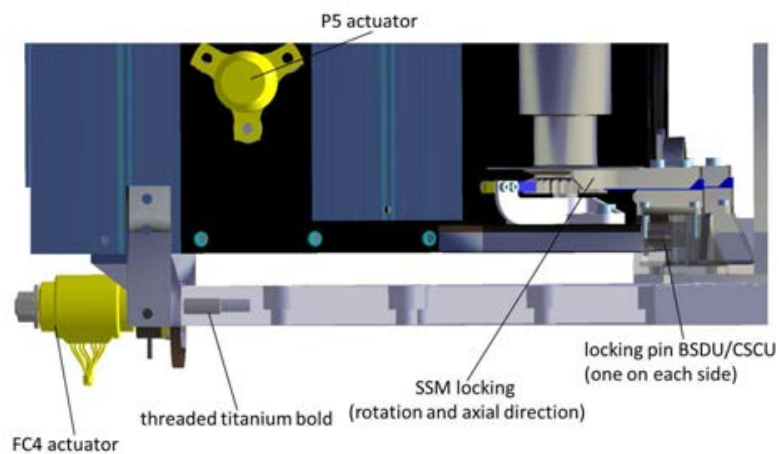

Figure 11: BSDU launch locks.

same as what is used for the sails, the $7.5 \mu \mathrm{m}$ polyimide film coated with aluminum. Low reflective segments consist of nearly transparent polyimide sections where the aluminum coating was removed. Using an optoelectronic sensor, it is possible to determine position based on counts of changes in reflectivity. Implementing periodically occurring calibration markers and a slight phase shift in the pattern (not shown in Figure 12) provides higher robustness of position determination.

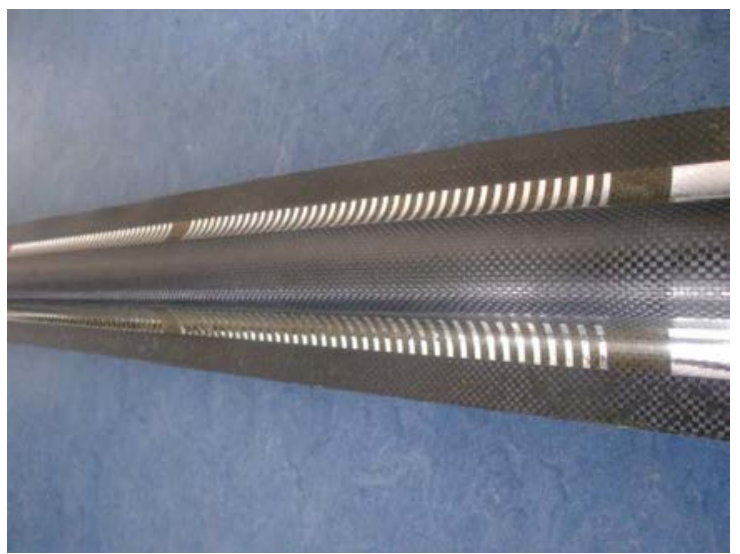

Figure 12: Reflective marker on the boom. An optical sensor determines the deployment process by scanning the markers.

\subsubsection{Camera system for visual documentation}

For the Gossamer-1 camera system, commercial off-theshelf (COTS) components are used. The chosen camera is a VRmagic VRmDC-8 Pro (resolution of $2056 \times 1544$ pixels, for details see data sheet VRmagic (2009)) with Edmund Optics NT68-672 lenses (see data sheet Edmund (2015)) for wide field and Pentax H1214-M lenses (see data sheet Pentax (2003)) for telemetric application. The camera is based on an industrial standard for machine vision using the C-Mount lens adapter system. This is a robust standard with a wide selection of different lens systems with different apertures and focal length. The camera housing was redesigned according to the expected mechanical loads. 
The camera system consists of 9 cameras. One camera is placed on each deployment unit (Figure 9(g) , facing inwards and covering the full spacecraft by wide field optics. Three cameras on the central unit face outwards with wide field optics, thereby covering one sail segment and adjacent booms and deployment units. Two of these are positioned such that stereoscopic viewing is supported. The two remaining cameras on the central unit use telemetric field optics and cover one boom and its deployment unit with a depth of field ranging from roughly the initial unlocking up to the final position.

Image data volume acquired by the camera system is limited by taking sequences of images rather than videos. Image data volume is decoupled from deployment speed by taking one image per $2.5 \mathrm{~cm}$ of deployment distance, as measured along the booms. Image data volume is further limited by taking sequences of images consisting of patterns of a number of low resolution images always followed by one high resolution image. Furthermore, on-board post processing and corresponding processor work load on the on-board computer is avoided as the camera allows resolution settings and definition of Regions of Interest (ROI) via hardware settings.

\subsubsection{Strain gauges}

During deployment, the boom loads are determined by strain gauges attached to the booms near the BSI as shown in Figure 13. Besides general monitoring for an onorbit characterization of the deployment system, they are also used to detect boom overload, which would trigger an emergency stop and additional FDIR. Zander et al. (2014) presented preliminary experiments for future in-orbit load and deflection monitoring.

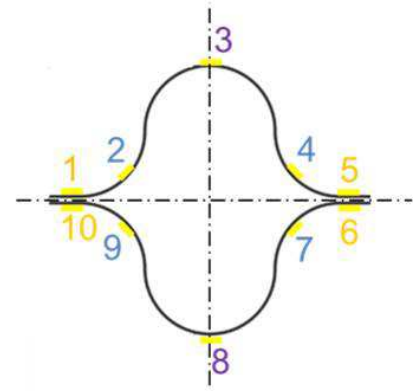

(a)

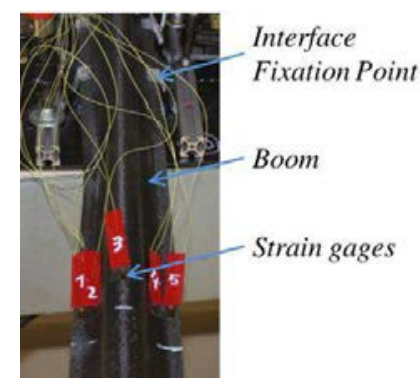

(b)
Figure 13: Gossamer-1 BSI with strain gages.

\section{Development testing of the deployment technol- ogy}

In order to validate the technology, a series of development as well as qualification tests were performed employing different engineering models. The tests ranged from mechanical characterization of booms, sail manufacturing and sail folding techniques, to characterization and functional testing of individual mechanisms up to a full two- segment system level deployment test under ambient conditions. Qualification testing of one deployment unit with boom and adjacent sail segments will be carried out with the qualification model currently being built. This also includes all electronic subsystems contained in the deployment unit.

\subsection{Boom testing}

Booms were tested in a vertical test rig (Figure 15) for bending, lateral and compression loads. In previous investigations, booms were only tested with an ideally stiff clamping at the boom mounting point. In contrast, for the present tests, booms were attached to the flexible BSI to provide realistic elasticity of the mounting. The boom was mounted vertically hanging from the BSI so that gravitational effects only affected the less sensitive axial load cases.

Different boom lengths were tested at different angles of attack. Loads were applied at the tip of the boom by a rope and pulley setup as shown in Figure 15. The angle of attack was varied by suitable positioning of the adjustable pulley. During a test run, the angle of attack changes slightly due to the deflection of the boom. This needs to be considered in the theoretical analysis.

Boom tip deflections was measured in all three degrees of freedom with laser triangulators and a photogrammetry system. Measuring the resulting force values was accomplished with force sensors on a fixed pulley within the test rig. All measured data were automatically acquired at a sampling rate of $9 \mathrm{~Hz}$. During testing, pulling force was steadily increased until stability failure occurred. When damage was not fatal, the test was repeated with the same boom to gain insight into effects of pre-damage on stability failure. Results for load at failure, i.e. maximum bearable load, as a function of angle of attack are shown in Figure 14. Bearable loads increase for a smaller angle of attack, reflecting the fact that axial load is less critical than lateral load. Straubel et al. (2014) presented further details about those tests.

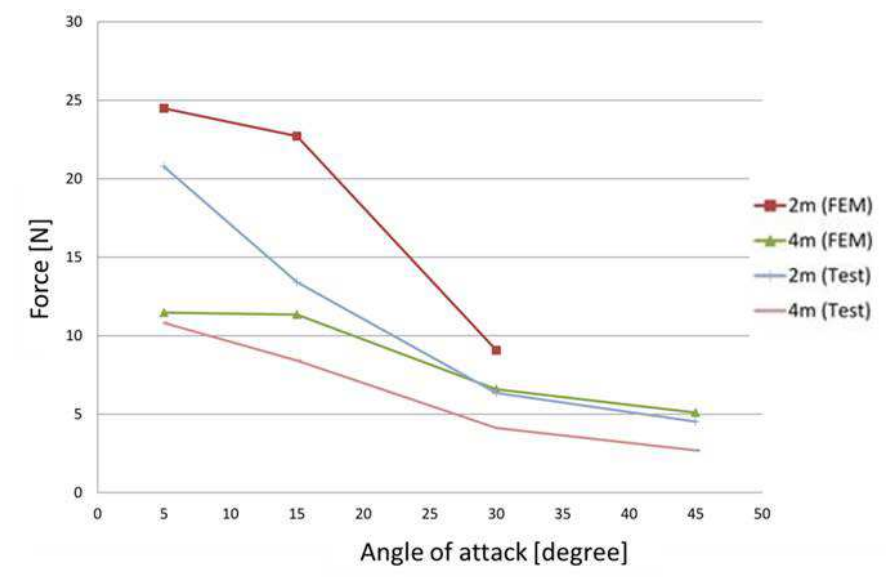

Figure 14: Load capacity of the booms for $2 \mathrm{~m}$ and $4 \mathrm{~m}$ length. The blue and pink graphs show the test results while the red and green graphs show the theoretical prediction. 
With increasing load, buckling deformation occurred at the flanges, qualitatively in line with FEM simulations. However, FEM results indicated greater stability than found in the experiment. This is considered to be an effect of differences in the clamping in the FEM and in reality, differences in the modeling of the material, and manufacturing imperfections of the tested specimens.

\subsection{Sail testing}

Pre-qualification tests were performed with a sail packaged according to Figure 6 and stowed on two sail spool engineering models. The sail was built as mechanically flight representative. Thin film photovoltaics were not included as such, but were simulated by suitable mechanically representative dummies made from flexible PCB material, i.e. copper-polyimid laminates of suitable dimensions (Figure 16). Harnessing on the sail was accomplished using loosely bundled high-quality industrial PTFE-insulated AWG28 litz wire. The wire type used was previously qualified at system level for MASCOT (see Ho et al. (2016)). Tests were performed as a representative cycle starting with a shaker test followed by centrifugal acceleration, fast decompression, and finally deployment under ambient conditions (see Subsection 5.3). The tests were also presented by Seefeldt et al. (2014b). Test loads were based on the launcher load envelope (Subsection 2.3).

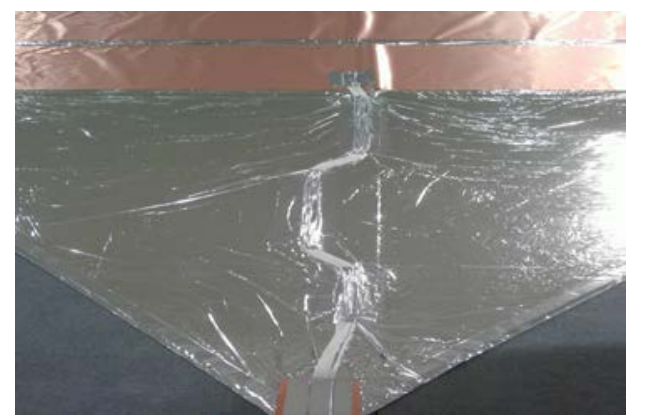

Figure 16: Sail with flexible PCB dummies and AWG 28 harness bundle.

All tests were conducted with a test adapter specially designed for those tests. The adapter is shown in Figure 17. Regarding the sail spools, these has a representative bearing and locking mechanism included. For final functional verification, the sail package and sail spools were transferred to the deployment test rig as described in Subsection 5.3.2. The Sail package and sail spools passed the test successfully with no anomalies observed.

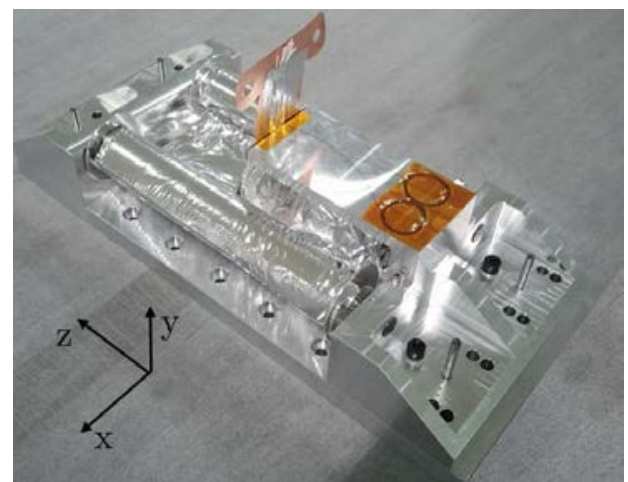

Figure 17: Sail package mounted on test adapter.

\subsection{Deployment testing}

Laboratory deployment tests were made at different levels of complexity, starting with individual subsystems up to final fully functional system level tests. The goal was the verification of

- the general deployment strategy,

- sail stowing and deployment,

- boom stowing and deployment,

- related force budgets,

- mechanism functionality,

- the electronics involved (position determination, camera system, on-board wireless communications),

- the deployment logic implemented.

Boom stowing and boom deployment was tested in socalled boom-pull out tests with just a single boom and a mechanically functional representative BSDU. No sails or sail deployment simulators were applied. Force sensors were used to determine force budgets (see Subsection 5.3.1).

As a last step of the sail testing as described in Subsection 5.2, one sail segment was deployed, employing linear drive units instead of booms and BSDUs. In a next step this test rig was extended in order to deploy two sail segments with one boom and one BSDU. This test setup also enables a system level test including mechanisms, electronics and implemented logic. These deployment tests are presented in Subsection 5.3 .2 .

\subsubsection{BSDU boom pull-out test}

To establish a deployment force budget (to be later translated into a motor and transmission torque budget, see below), as a first step, isolated boom pull-out tests were performed. In these tests, only boom and boom deployment related forces and frictions were measured. Figure 18 shows the basic set-up of the boom pull-out test. For this test, the belt is not wound up internally by the belt winding mechanism, but externally by a suitable rope 

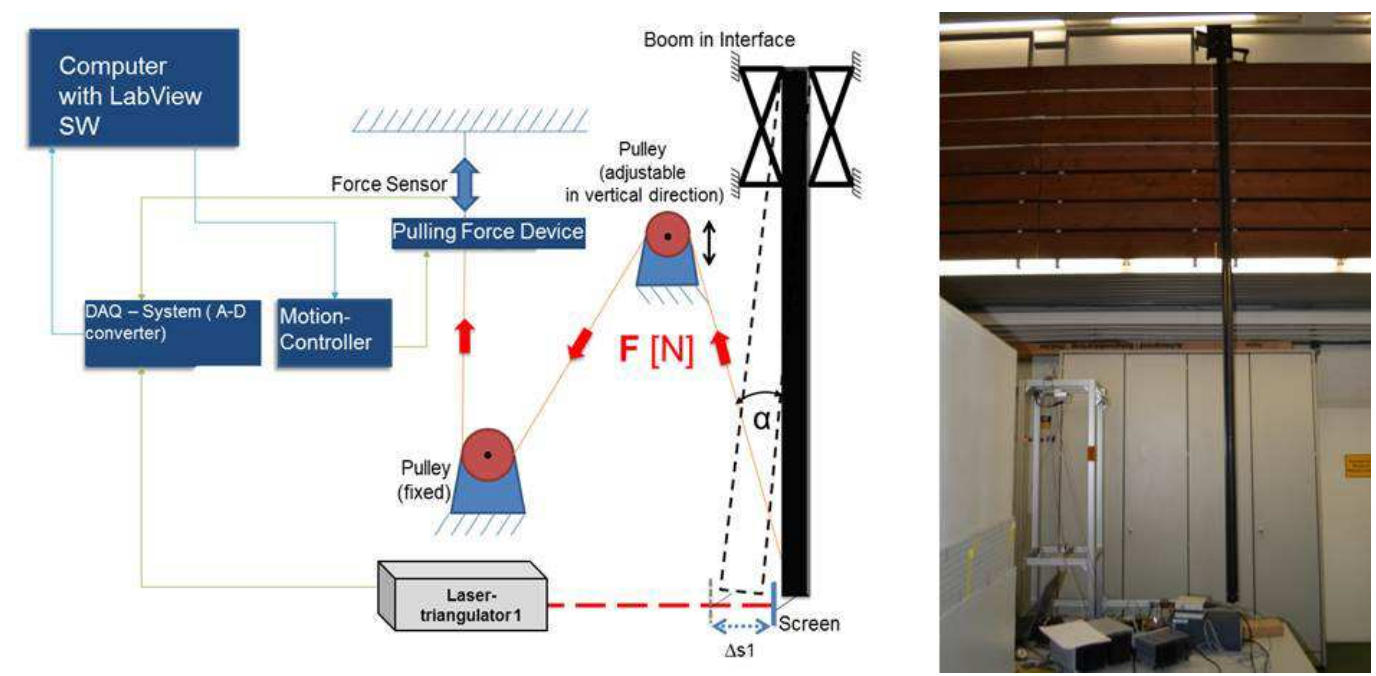

Figure 15: Boom bending tests.

and pulley arrangement including external motor and force sensor. Apart from the full BSDU configuration, including all friction inducing elements, reduced configurations were also measured, in which one friction inducing element after another was taken out step by step. This allowed a mathematical separation of contributions from individual friction elements.

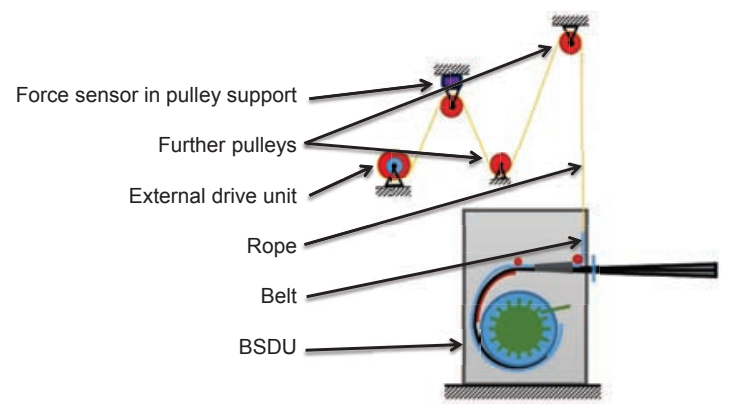

Figure 18: Test setup for belt force determination test.

Figure 19 shows results for the full BSDU configuration acquired at $5.5 \mathrm{~mm} / \mathrm{s}$ deployment speed. Different deployment speeds were tested, and this one is closest to the finally realized speed of $0.84 \cdot 10^{-2} \mathrm{~m} / \mathrm{s}$ (see Subsection 4.3.1). The influence of the difference in the deployment speeds on forces measured is considered to be negligible. High frequency oscillations in the signal are caused by the leaf spring of the boom hub brake mechanism. Low frequency variations are due to imperfections and inhomogeneities regarding the material and manufacturing of the boom. Values vary from roughly 5 to $15 \mathrm{~N}$ with an approximate average of about $10 \mathrm{~N}$.

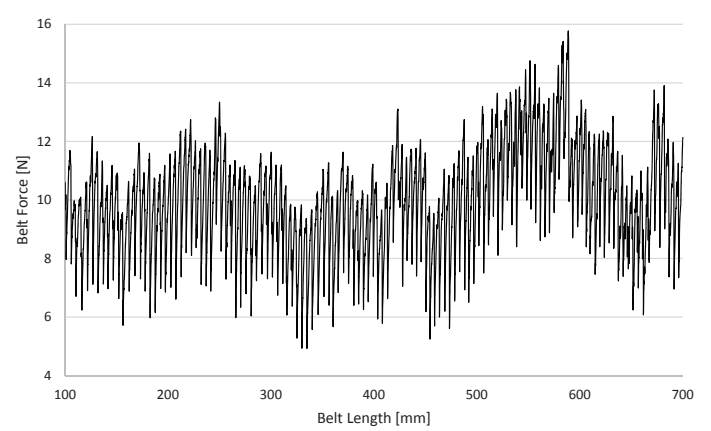

Figure 19: Boom deployment force of the BSDU engineering model without sail segments at $5.5 \mathrm{~mm} / \mathrm{s}$ deployment speed.

\subsubsection{System level deployment test}

Sail packaging and deployment as such was tested in a set-up without booms and BSDU. Two linear drives $(4.5 \mathrm{~m}$ maximum length each) at right angles with sail spools mounted on them were used to deploy one sail segment (Figure 20). Three-axes force sensors were placed between the linear drives and sail spools. Deployment took place via controlled movement of the linear drives. Movement was under computer control. Different deployment speeds as well as speed profiles could be tested. This test rig was then extended for a system level test with two sail segments and one boom.

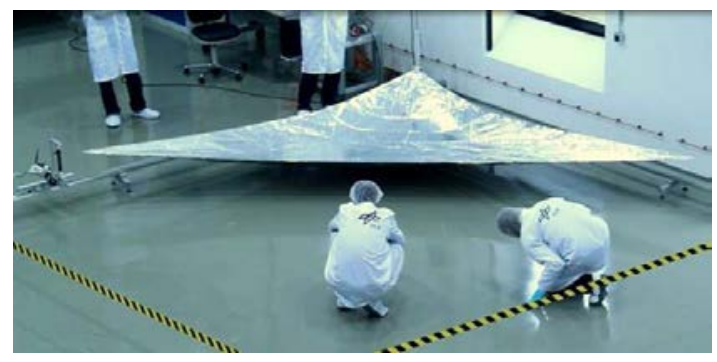

Figure 20: Deployment test of one sail segment alone without booms and BSDU. 


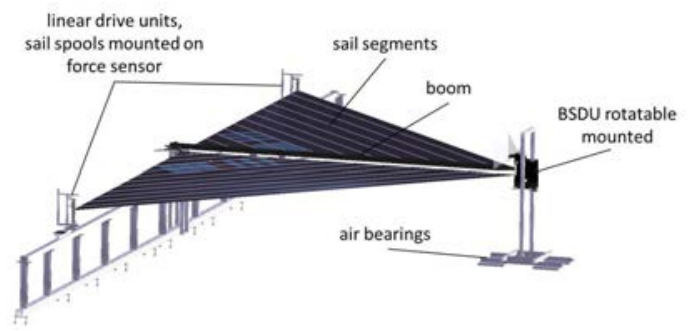

Figure 21: Test rig for the deployment of two sail segments together with one boom by employing the Gossamer-1 deployment mechanisms.

Fully functional system level tests used two linear drives arranged in a line simulating one full boom diagonal of a Gossamer-1 configuration with a boom and BSDU tested at right angles between them (Figure 21). The boom was mounted to a BSI and was deployed by a fully functional BSDU engineering model. The BSDU was supported by a test rig, which glide on air bearings to provide minimum friction in order to allow free BSDU and boom movement within the sail plane. On each linear drive, a sail spool was mounted on top of a three-axis force sensor. The BSDU was fully equipped including the sail spools at its sides. One boom and both adjacent sail segments could thereby be deployed in this setup, designated as one-boom-two-sailsegment system level testing. The BSDU was controlled by the on-board wireless communications with a fully functional CSCU electronics system as the counterpart, which in turn was connected to the electronic ground support equipment and suitable control interfaces. Acquisition of sensor data as well as BSDU camera images was implemented. Data was transferred via the on-board wireless communications system from the BSDU to the CSCU.

The deployment test with its different phases as described in Subsection 2.7 is shown in Figure 22. The deployment (Phase 4) is shown in Figure 22(a), followed by the separation (Phase 5) in Figure 22(b) and the BSDU jettison (Phase 8 ) in Figure 22(c). In addition, Figure 22(d) shows an image acquired by the BSDU on-board camera during a deployment test.

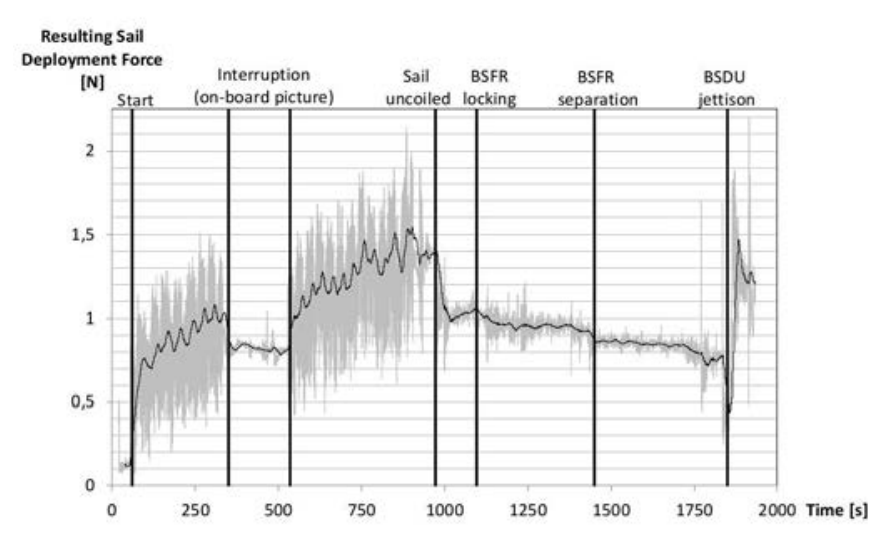

Figure 23: Measured deployment forces during laboratory deployment test. Measured with $12.5 \mathrm{~Hz}$, black graph represents the moving average for every 255 data points.

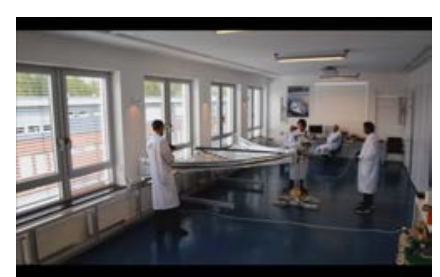

(a) Deployment (Phase 4) and acquisition of picture with on board camera (Figure 22(d).

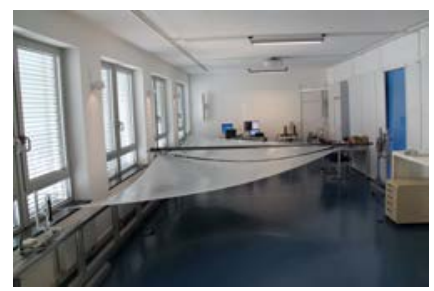

(c) BSDU jettison - Phase 8 .

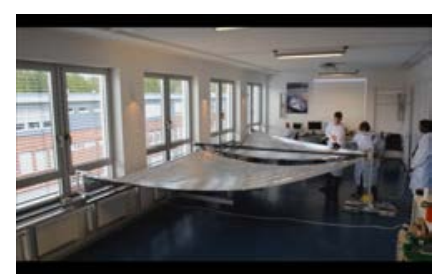

(b) Sail completely deployed, BSDU separation - Phase 5.

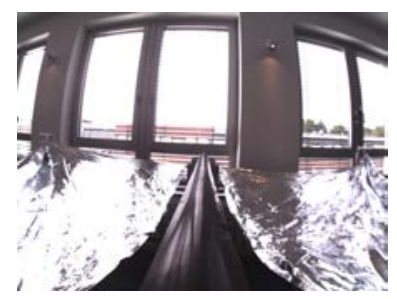

(d) Picture taken with the onboard camera during the deployment.
Figure 22: Gossamer-1 deployment test in progress.

In the same way as in the boom pull-out, the sail spools and sail deployment also introduce additional forces when pulling off the sail from the sail spool. These forces were measured during deployment by force sensors placed between the two linear drive units and their corresponding sail spool mounts. Data was recorded as a function of time, and thereby as a function of deployment phases ranging from stowed configuration up to final jettison of the BSDU. Results obtained for one of these sensors are shown in Figure 23. Maximum values were observed at 2.2 N. This applies to one sail spool. As one BSDU has to pull off sail halves of two sail spools, this force must be considered twice in the budget, resulting in $4.4 \mathrm{~N}$.

Similar to the boom pull-out, the sail pull-off measurements also showed a fast oscillation caused by the leaf spring brake at the sail spool. The oscillations are roughly 
Table 2: Overview of the torque budget for the motor sizing. Translating the force budget to a torque budget considers the BWM diameter of $40 \mathrm{~mm}$. Uncertainty and design factors according to the ECSS (2009) standard are applied.

\begin{tabular}{|l|l|l|l|}
\hline Component/Type & Torque [Nm] & Uncertainty Factor & Design Torque [Nm] \\
\hline \hline Boom Hub Brake / Spring & 0.1034 & 1.2 & 0.1241 \\
\hline CFRP-Shell / Friction & 0.0418 & 1.5 & 0.0627 \\
\hline Ramp/Boom / Friction & 0.0356 & 1.2 & 0.0427 \\
\hline Drum Friction & 0.0288 & 1.5 & 0.0432 \\
\hline BSFR / Friction & 0.0264 & 1.5 & 0.0396 \\
\hline Sail Spool Brake / Spring & 0.0520 & 1.2 & 0.0624 \\
\hline Sail Deployment / Gravity, Friction & 0.0360 & 1.5 & 0.054 \\
\hline Inertia & 0.0020 & 1.1 & 0.0022 \\
\hline \hline Sum & 0.3260 & $(1.3)^{\mathrm{a}}$ & 0.4309 \\
\hline \hline Final Design Torque including a safety factor of 2 & 0.8618 \\
\hline
\end{tabular}

${ }^{a}$ Resulting overall uncertainty factor.

about 1.3 N. Besides a smaller amount of friction between the tip of the spring and the gear, this mainly represents the deformation of the spring. The general trend of an increasing force during the sail deployment is due to two main effects. The diameter of the sail coiled on the spool is changing. At the beginning, the diameter of the coiled sail is about $50 \mathrm{~mm}$, and at the end it is the spool diameter of $35 \mathrm{~mm}$. This in turn leads to a force increase of about $0.4 \mathrm{~N}$. In addition, the deployed sail introduces tension forces, especially when deployed in the laboratory under gravitation. The highest forces appear at the point shortly before the sail is completely deployed and when jettisoning the BSDU. At both times the force is about $2.2 \mathrm{~N}$.

The oscillating force is present up to the point where the BSFR is locked into the boom, which, due to the design, occurs just after having pulled the sails completely off of the sail spools. When the last tip of the sail segment slips off of the spool, the sail gets a little more slack. As the sail is less tensioned at that point, a decrease of approximately $1 \mathrm{~N}$ appears. After having pulled off the sail completely, only more or less constant force is observed, which is equal to the tension force applied to the sails. After the separation of the BSDU from the BSFR, the force measured reduces slightly, indicating that due to the additional flexibility of the BSFR interface, the sail tension is also slightly reduced. Final and stronger oscillations towards the end of the measurement are caused by perturbations due to the BSDU rolling off of the boom (jettisoning).

\subsubsection{Deployment force budget results and motor sizing}

Figure 24 shows the measured contributions to the full force budget.

Forces purposely added by the sail spool and boom hub leaf spring brake add up to $5.2 \mathrm{~N}+2.6 \mathrm{~N}=7.8 \mathrm{~N}$, whereas the major friction related contributions of the boom deployment add up to $6.6 \mathrm{~N}$. In addition, the laboratory sail deployment introduces forces of $1.8 \mathrm{~N}$. This is mainly gravitation related, but also includes an unknown fraction of

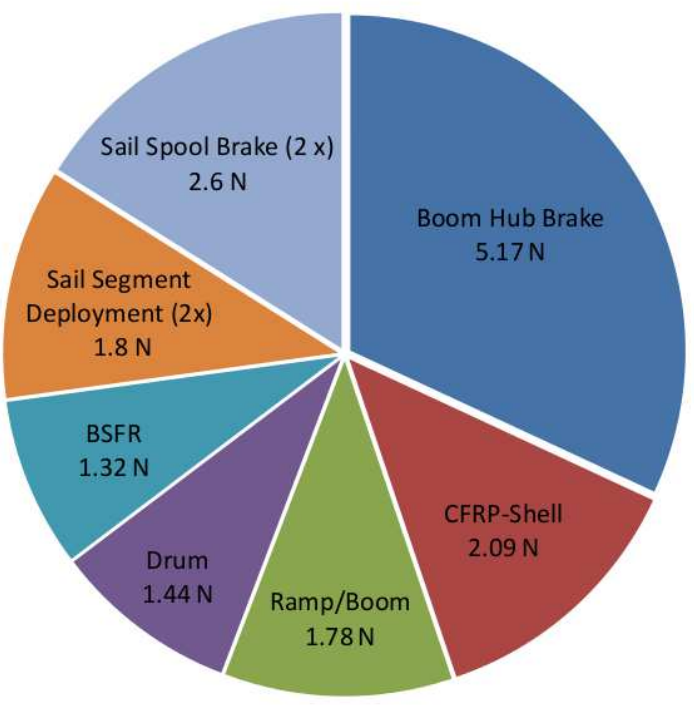

Figure 24: Composition of the deployment force that is introduced through the BWM (see section 4.3.1).

friction force of the sail spool mechanisms. These forces transform into the required drive torque at the gear's axis through multiplication with the radius of the belt winding mechanism's spool of $20 \mathrm{~mm}$. The torques were multiplied with uncertainty and safety factors according to the ECSS (2009) standard. Table 2 provides an overview of the torque budget for the motor.

\section{Conclusion}

A deployment strategy was invented for a mission that aims to demonstrate a controlled and autonomous deployment in LEO. For this deployment strategy, a bus system as well as the required deployment mechanisms and electronics were developed, and engineering models of the hardware were built.

Deployment on system level was successfully demonstrated to be robust, controllable, and at no time at risk 
of entangling. The latter is guaranteed by the folding concept, which ensures that at each stage of deployment, only a minimum amount of the sail is released. The boom, BSI and BSDU proved to be suitable to drive the combined deployment of booms and sails together. No negative interference between boom deployment and sail deployment was observed. The functionality of all mechanisms involved was demonstrated. The functionality of the electronics involved was also demonstrated, i.e. wireless control, deployment logic implemented, data acquisition, image acquisition by the on-board camera (see Figure 22(d) and a ground segment representative control via the electronics ground support equipment.

The deployment technology is on TRL four approaching level five, with a qualification model for environmental testing currently being built.

The qualification model of the deployment unit will be subjected to environmental testing. A sequence of tests will be performed including fast venting, vibration testing, thermal-vacuum testing and laboratory deployment. The development of solar sail specific technology will be stopped after the qualification process on a TRL level five until further political support and funding can be acquired. The further development of deployment technology will focus on huge solar arrays possibly based on thin-film photovoltaics.

\section{Acknowledgments}

This work was funded by DLR German Aerospace's Research and Development program for technology of space systems as "Gossamer-1 Deployment Technology Demonstrator Project". The launch opportunity was funded by EC-Project "QB50", Project Identifier 284427, FP7-SPACE2011-1, "QB50-An international network of 50 CubeSats for multi-point, in-situ measurements in the lower thermosphere and re-entry research". The authors appreciated the fruitful and constructive cooperation with all QB50 and EC representatives.

The authors wish to express their gratitude for excellent technical discussions, review support and advice by ESA/ESTEC-TEC-M and D/TEC-MTT. Regarding solar sail specific mission design the authors are indebted to ESA ESTEC TEC-M for organizational support and to Bernd Dachwald (University of Applied Sciences Aachen), Malcolm Macdonald (University of Strathclyde) and Collin McInnes (University of Glasgow) for leading three independent solar sail candidate mission studies. Furthermore, the authors want to thank their project partners at University of Würzburg, Aerospace Information Technology as well as at RWTH Aachen, Institute of High Frequency Engineering for fruitful cooperation and discussion.

And finally our thoughts are with late Rüdeger Reinhard, together with Jean Muylaert from VKI Brussels one of the godfathers to QB50 and Gossamer-1 within QB50. As ESA Consultant, Ruedeger's everlasting initiative and diplomacy brought people together, brought projects into existence and without him this project would never have reached the stage of maturity, which it has reached by now, no matter, which obstacles still pertain. He inspired people and created visions. The world needs more people of this kind. Instead, we grieve for him, having one less of his kind among us. 
Appendix A. Gossamer-1 Parts

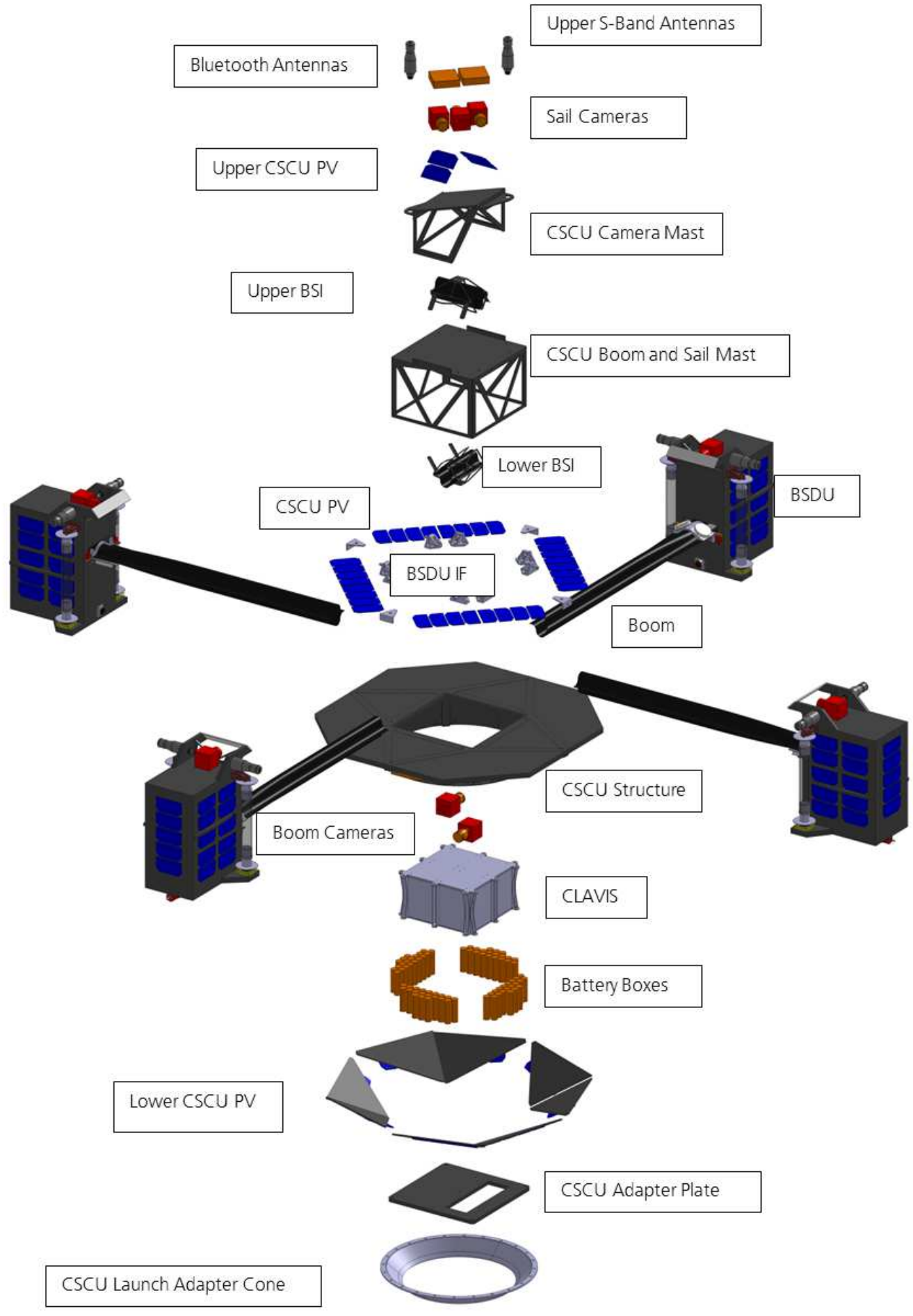

Figure A.25: Gossamer-1 exploded view. 


\section{Appendix B. Launch Loads}

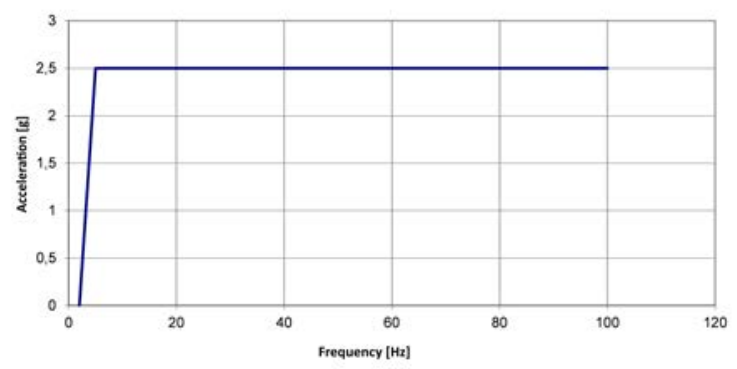

Figure B.26: Lateral sine vibration loads derived from launcher envelope.

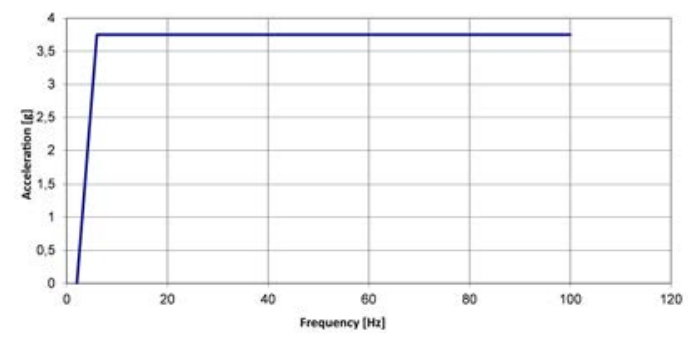

Figure B.27: Longitudinal sine vibration loads derived from launcher envelope.

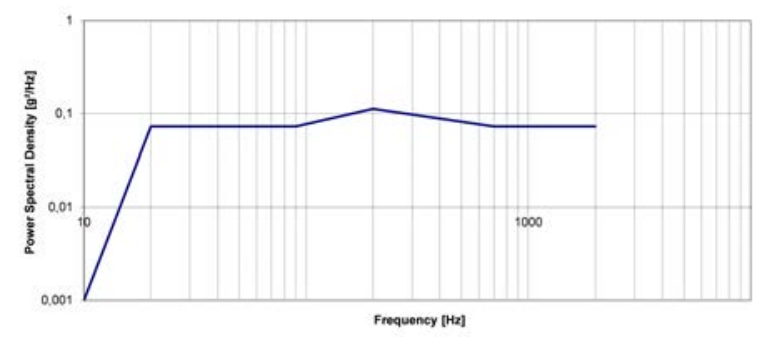

Figure B.28: Random vibration loads derived from launcher envelope $\left(G_{r} m s=12.4\right)$.

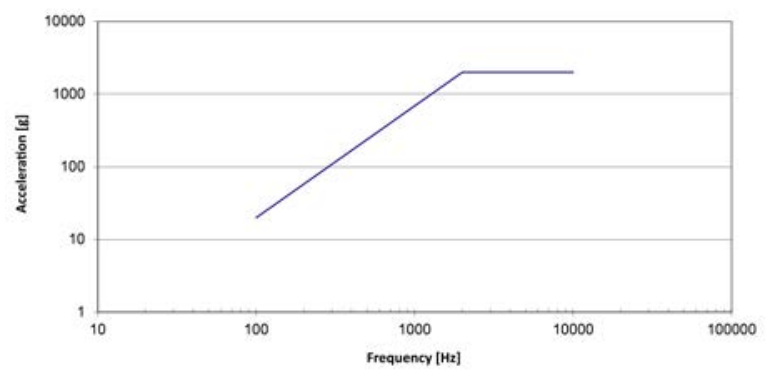

Figure B.29: Shock loads derived from launcher envelope. 


\section{Appendix C. Electronic Schematic}

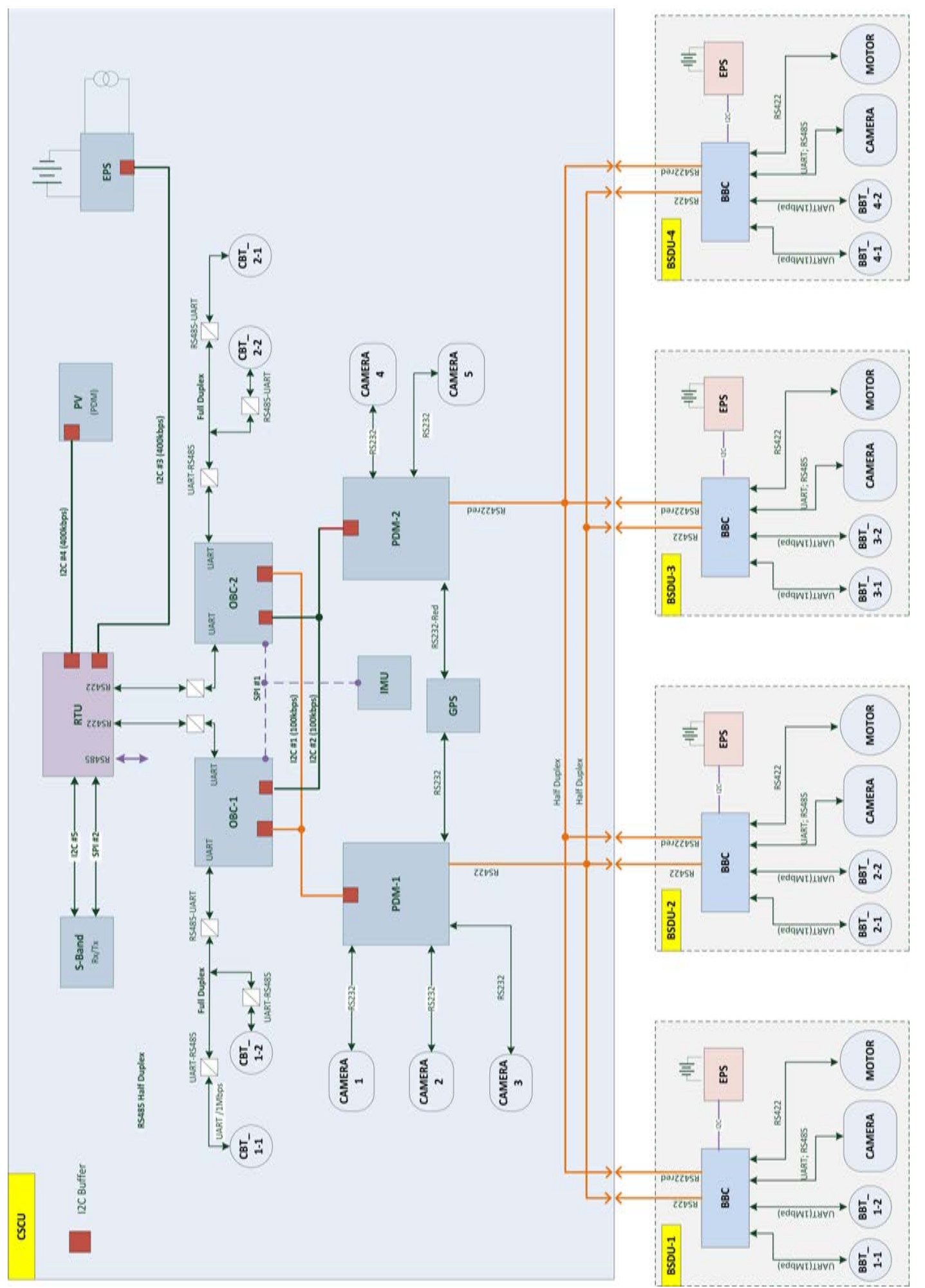

Figure C.30: Gossamer-1 bus schematic. 


\section{Appendix D. Eigenfrequencies}

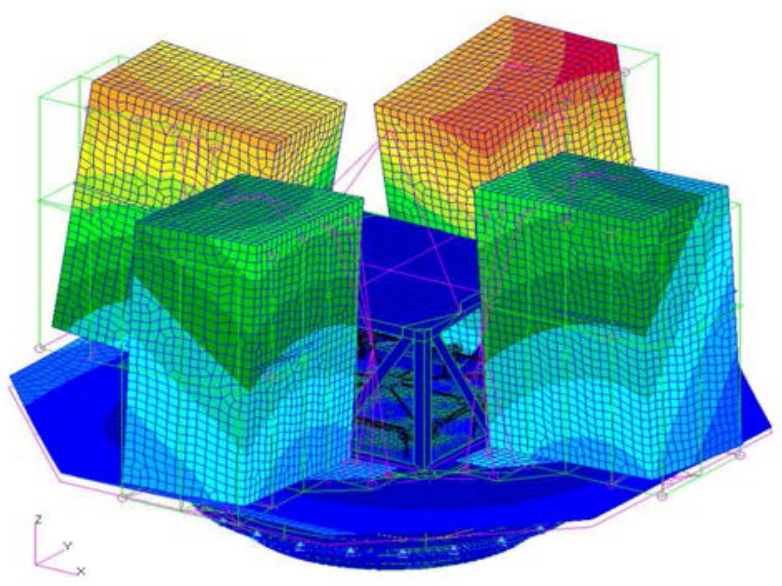

(a) Mode $1,106 \mathrm{~Hz}$

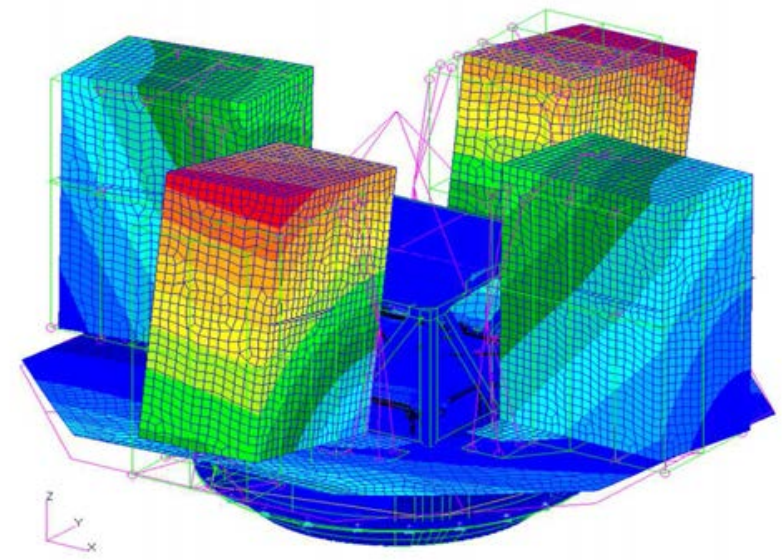

(b) Mode 2, $107 \mathrm{~Hz}$

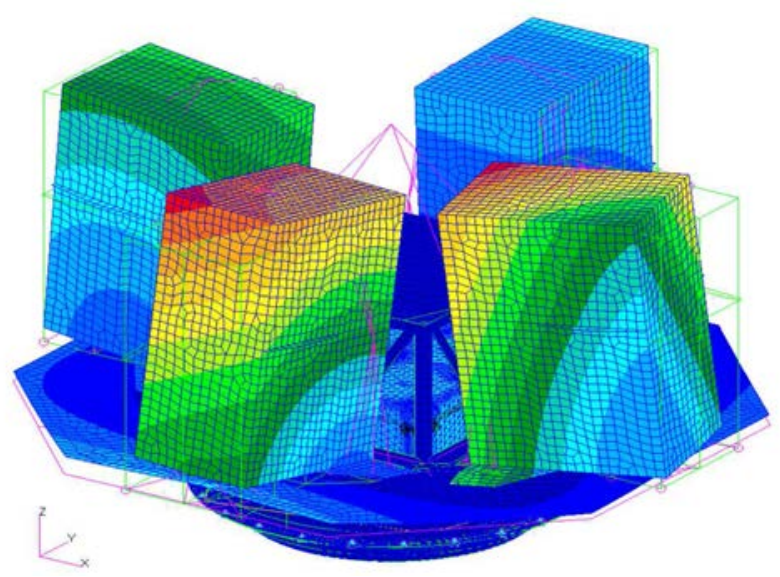

(c) Mode $3,125 \mathrm{~Hz}$

Figure D.31: Deformation plots of the first three eigenmodes with a modal mass of more than $10 \%$. Blue areas are undeformed while red areas show the highest deformation.

\section{References}

Leipold, M., Garner, C. E., Freeland, R., Hermann, A., Noca, M., Pagel, G., Seboldt, W., Sprague, G. \& Unckenbold, W., ODISSEE - A proposal for demonstration of a solar sail in earth orbit, Acta Astronautica 45/4, 557-566, 1999.

Leipold, M, Eiden, M., Garner, C. E., Herbeck, L., Kassing, D., Niederstadt, T., Krüger, T., Pagel, G., Rezazad, M., Rozemeijer, H., \& others, Solar sail technology development and demonstration, Acta Astronautica 52/2, 317-326, 2003.

Leipold M., Widani C., Groepper P. \& others, The European Solar Sail Deployment Demonstrator Mission, Proceedings of the International Astronautical Congress, 2006.

Agnolon D., Study overview of a solar sail demonstrator: GEOSAIL, DLR/ESA, 2008

Tsuda Y., Mori O., Funase R., Sawada, H., Yamamoto, T., Saiki, T., Endo, T., Yonekura, K., Hoshino, H. \& Kawaguchi, J., Achievement of IKAROS - Japanese deep space solar sail demonstration mission, Acta Astronautica, 82/2, 183-188, 2013.

Johnson, L., Whorton, M., Heaton, A., Pinson, R., Laue, G. \& Adams, C., NanoSail-D: A solar sail demonstration mission, Acta Astronautica 68/5, 571-575, 2011.

Ho, T., Baturkin, V., Grimm, C., Grundmann, J. T., Hobbie, C., Ksenik, E., Lange, C., Sasaki, K., Schlotterer, M., Talapina, M., \& others, MASCOT - The Mobile Asteroid Surface Scout Onboard the Hayabusa2 Mission Space Science Reviews, 1-36, 2016.

Spröwitz, T., Bauer W., Drobczyk M. \& others, CLAVIS - Erste Schritte zu einer standardisierten NanoSat-Platform, Proceedings of the DGLR Kongress, 2010.

Zander, M. E., Sinapius M, \& Hühne, C., Preliminary Experiments for an On-Orbit Detection system to Monitor Load and Deflection States of Thin Shell CFRP Booms for the Solar Sail Demonstrator Gossamer-1, Proceedings of the European Conference on Spacecraft Structures, Materials and Environmental Testing, 2014.

Straubel, M., Seefeldt, P., Spietz, P. \& Hühne C., The Design and Test of the GOSSAMER-1 Boom Deployment Mechanisms Engineering Model, Proceedings of the 2nd AIAA Spacecraft Structures Conference, 2015.

Seefeldt, P., Steindorf, L. \& Spröwitz, T., Solar Sail Membrane Testing and Design Considerations, Proceedings of the European Conference on Spacecraft Structures, Materials \& Environmental Testing, 2014.

Seefeldt, P., Spietz, P. \& Spröwitz, T., The Preliminary Design of the GOSSAMER-1 Solar Sail Membrane and Manufacturing Strategies, Advances in Solar Sailing, 133-151, 2014.

Seefeldt P.\& Spietz, P., Raumfahrzeug-Membranentfaltungssystem und Verfahren zum Betrieb desselben, Pending Patent 2016

Straubel, M., Zander, M. \& H'uhne, C., Design and Sizing of the GOSSAMER Boom Deployment Concept Advances in Solar Sailing, 593-608, 2014

Thoemel, J., Singarayar, F., Scholz, T. \& others, Status of the QB50 CubeSat Constellation Mission, Proceedings of the 65th International Astronautical Congress, 2014.

ECSS-E-ST-01C, The European Cooperation for Space Standardization, 2009.

ISO24113 Space systems, Space debris mitigation requirements, International Organization for Standardization, 2011.

VRmDC-8 PRO (color), VRmagic, 2009.

Techspec Fixed Focal Length Lens Specifications, Edmund Optics, 2015.

For Machine Vision H1214-M, 12 mm 1:1.4, Pentax Optics, 2003. 